\title{
Interpretation of freezing nucleation experiments: singular and stochastic; sites and surfaces
}

\author{
G. Vali \\ University of Wyoming, Department of Atmospheric Science, Laramie, WY 82070, USA \\ Correspondence to: G. Vali (vali@uwyo.edu)
}

Received: 31 December 2013 - Published in Atmos. Chem. Phys. Discuss.: 20 January 2014

Revised: 14 April 2014 - Accepted: 15 April 2014 - Published: 2 June 2014

\begin{abstract}
Publications of recent years dealing with laboratory experiments of immersion freezing reveal uncertainties about the fundamentals of heterogeneous freezing nucleation. While it appears well accepted that there are two major factors that determine the process, namely fluctuations in the size and configuration of incipient embryos of the solid phase and the role of the substrate to aid embryo formation, views have been evolving about the relative importance of these two elements. The importance of specific surface sites is being established in a growing number of experiments and a number of approaches have been proposed to incorporate these results into model descriptions. Many of these models share a common conceptual basis yet diverge in the way random and deterministic factors are combined. The divergence can be traced to uncertainty about the permanence of nucleating sites, to the lack of detailed knowledge about what surface features constitute nucleating sites, and to the consequent need to rely on empirical or parametric formulas to define the population of sites of different effectiveness. Recent experiments and models, consistent with earlier work, demonstrate the existence and primary role of permanent nucleating sites and the continued need for empirically based formulations of heterogeneous freezing. In order to clarify some aspects of the processes controlling immersion freezing, the paper focuses on three identifiably separate but interrelated issues: (i) the combination of singular and stochastic factors, (ii) the role of specific surface sites, and (iii) the modeling of heterogeneous ice nucleation.
\end{abstract}

\section{Introduction}

In order to clarify some aspects of immersion freezing nucleation, the results of laboratory experiments and their interpretations in terms of models are examined in this paper. Many such experiments have been reported in recent literature. Disagreements about some of the fundamentals of the process continue to present problems, yet a considerable degree of commonality in the data can also be identified. Both sides of the situation will be examined. Immersion freezing, or simply freezing, refers here to the formation of ice from liquid water via nucleation by solid impurities in the liquid. Independent of the type of solid surface involved, they are labeled ice nucleating particles (INPs) in this paper.

It is well known that heterogeneous nucleation of ice takes place at quite different temperatures, depending on the chemical and physical properties of particles present in the water, or in contact with it. It is also fairly well established that the probability of nucleation increases in proportion to the total amount, more specifically the total surface area of the particles. On the other hand, still little is known about the specific surface properties that govern what conditions lead to ice nucleation and about the structure of the ice embryos which eventually become stable and grow to macroscopic ice. Conceptual descriptions of heterogeneous nucleation center on two main factors: one factor specific to the interaction between the nucleating surface and the embryo of the new phase, the other due to random timing of the formation of a stable embryo. The first can be viewed as a static factor, the second as a dynamic one. The static factor is very complex because it involves details about what surface properties or anomalies serve as sites for embryo formation. In addition, it has to incorporate details about how the sites are distributed among otherwise identical particles, and how particles are 
distributed among sample volumes of water in an experiment. The first of these difficulties is by far the most serious and is the fundamental reason why theoretical descriptions remain rough approximations.

The main elements of the dynamic factor can be envisaged in terms of the energy difference between the solid and liquid phases and the rate at which molecules are added to the embryo or detach from it. This view has been confirmed for homogeneous nucleation. Only rough estimates are available for defining the same parameters for a crystalline embryo on a substrate. Classical nucleation theory (CNT) is based on the foregoing description with two assumptions, namely that the embryos can be viewed similarly to liquid drops and that the interaction with the foreign surface can be characterized in terms of the contact angle between that surface and water. These assumptions are considered substitutes for more definitive parameters until those are identified.

In accord with the two types of factors discussed, emphasis in experimental studies is placed on measurements of the temperature dependence and/or of the time dependence of nucleation. Interpretations of the experimental results face considerable difficulties in identifying the relative importance of the two factors. At the most basic level, the difficulty arises from the impossibility to fully decouple temperature and time in any practical experimental approach. This dilemma gave rise very early in the history of freezing nucleation studies to two separate ways of interpreting the same observations; one assigned primacy to time the other emphasized the temperature-dependent impacts of the impurities. The origins of this dichotomy - the stochastic and singular models - are reviewed in Appendix A.

A key question to examine is how the singular and stochastic aspects of heterogeneous freezing nucleation are evidenced in experiments, the models that have been constructed to describe that behavior, and how the evidence leads to models that combine both aspects. While given experiments often direct attention to one factor or another due to the design of the experiment, the reported results are actually more complementary than contradictory and can be interpreted in a consistent way, leaning on the relatively small impact of time-dependent effects.

\section{Singular vs. stochastic nucleation}

The quantitative statement of the singular model starts with assigning a "characteristic temperature" to each nucleating site and expresses the abundance of sites of various characteristic temperatures in terms of differential and cumulative nucleus spectra (Vali, 1971; hereafter V71; Murray et al., 2012; hereafter Mu12). The stochastic model is analogous to firstorder chemical kinetics; the principal parameter is the rate of nucleation as a function of temperature (e.g., Mu12).

In order to reduce ambiguities, it is important to note that there are at least three levels of random contributions to freezing experiments with distributed samples. The basic one is that resulting from molecular fluctuations of the ice embryo. This is clearly a stochastic process. The allocation of ice nucleating particles (INPs) in the sub-samples, as for example from a water sample in which INPs are suspended and subsequently divided into drops, is expected to be random (not stochastic). A possible third level of randomness arises from the location of the nucleating sites on the surfaces of the INPs. The principal use of the term "stochastic" in this paper is to refer to the molecular fluctuations of the embryos. The two other processes mentioned are random events and do not involve time sequences.

\subsection{Freezing rate vs. nucleation rate}

The time dependence expected in heterogeneous ice nucleation experiments depends on whether the singular or stochastic description is adopted. Predictions differ clearly for the two scenarios and the empirical results should dictate which of the two views is appropriate. However, many experiments do not contain tests of the critical measures, and, on the basis of features for which the two interpretations do not differ, arrive at conclusions not supported by the results of the specific experiment. Examination of experiments in this paper will focus on this issue and will do so with reliance on the distinction between "freezing rate" and "nucleation rate" as defined in the following paragraphs.

Freezing rate, $R$, is derived from observations of a number of externally identical ${ }^{1}$ sample units and is defined as the fraction of sample units freezing per unit time. It is a simple representation of observations and expresses the combined effects of the distribution of nucleating particles/sites within the sample units, the time dependence of nucleation and other possible factors deliberately introduced by experimental conditions. External parameters influencing the freezing rate are the sample volume and the time-temperature history to which the sample is exposed. Freezing rate is a ready representation of observations and is easily calculated when the sample units are identical with respect to these external parameters and all units originate from the same bulk sample. Weighted averages of the freezing rate, when the sample units are of different volumes but from the same bulk sample, can also be constructed but will not be introduced here (see V71 Appendix).

The freezing rate for $N_{0}$ samples at time $t$ when $N_{\mathrm{F}}$ units have frozen and $N_{\mathrm{L}}=\left(N_{0}-N_{\mathrm{F}}\right)$ have not yet frozen is defined $^{2}$ as

$R(t)=-\frac{1}{N_{\mathrm{L}}(t)} \frac{\mathrm{d} N_{\mathrm{L}}}{\mathrm{d} t}=\frac{1}{N_{0}-N_{\mathrm{F}}} \frac{\mathrm{d} N_{\mathrm{F}}}{\mathrm{d} t}$.

The negative sign is needed in the first form of the equation since $\mathrm{d} N_{\mathrm{L}}$ is negative. It is also possible to write Eq. (1) in

\footnotetext{
${ }^{1}$ The phrase "externally identical" refers to a set of sample units of the same volume and drawn from the same bulk sample.

${ }^{2}$ List of symbols and acronyms is at the end of the text.
} 
terms of the fraction of samples not frozen $g=N_{\mathrm{L}} / N_{0}$, or in terms of the fractions frozen $f=N_{\mathrm{F}} / N_{0}$ :

$R(t)=-\frac{1}{g(t)} \frac{\mathrm{d} g}{\mathrm{~d} t}=\frac{1}{1-f} \frac{\mathrm{d} f}{\mathrm{~d} t}$.

From Eq. (1), at a fixed temperature, and assuming $R_{T}(t)=$ const. (i.e., independent of time), the well-known expressions follow:

$\ln \left[N_{\mathrm{L}}(t) / N_{0}\right]=-R_{\mathrm{T}} \cdot t \quad$ and $\quad N_{\mathrm{L}}(t)=N_{0} \exp \left(-R_{\mathrm{T}} \cdot t\right)$.

These equations demonstrate an exponential decay in the number of unfrozen samples with time, at a rate defined by the constant $R_{\mathrm{T}}$. However, as it will be shown, the condition $R_{\mathrm{T}}=$ const. is rarely realized empirically.

A change to temperature dependence is readily made for experiments in which the samples are cooled at a rate $w=\mathrm{d} T / \mathrm{d} t$ (a negative number):

$R(t)=-w \cdot R^{*}(T) \quad$ and $\quad R^{*}(T)=-\frac{1}{N_{\mathrm{L}}(T)} \frac{\mathrm{d} N_{\mathrm{L}}}{\mathrm{d} T}$.

$R^{*}(T)$ can also be expressed in terms of $f$ or $g$ as above. In the simplest case, $w$ is a constant but the definition applies equally well if $w$ varies with time. It should be noted that $R^{*}(T)$ has dimensions of inverse temperature, while $R(t)$ has dimensions of inverse time.

Nucleation rate, $J$, is the probability of nucleation per unit time for a population of identical ice embryos. This quantity is derived in classical nucleation theory (CNT) by considering the rate of addition of molecules to the embryo and the assumed shape of the embryo. From an experimental perspective, the nucleation rate for homogeneous nucleation with all sample units of identical volume is equal to the freezing rate per unit volume of liquid:

$J_{\text {hom }}=J_{\mathrm{v}}=\frac{R}{V}$.

Similarly, the nucleation rate for heterogeneous nucleation is best defined as the probability of nucleation per unit surface area of substrate, $J_{\mathrm{s}}$. The main problem in applying this concept is how to envisage the existence, and how to ascertain empirically, identical embryos and the properties of the surface on which they form, so that the rate expression might be validly defined. The underlying problem is the lack of knowledge of what nucleating sites are and the absence of empirical methods of identification of the those sites. In the strictest sense, the nucleation rate could be determined with identical nucleating sites located in separate sample units (drops), or with a single site which can be repeatedly tested at identical conditions. Many authors extend the definition using the assumption that any part of the surface has an equal likelihood of containing a nucleating site or has the same probability distribution of nucleating sites of different effectiveness. The conversion from freezing rate is, in principle, via the expression

$J_{\mathrm{s}}=\frac{R}{A}$,

but the application of Eq. (6) is only justified if the conditions mentioned are fulfilled. That is, it is necessary to ensure that all sample units (drops) contain the same amount of substrate surface. This is achievable in practice. However, the validity of the assumption of uniform surface properties with respect to the probability of nucleation is debatable, and will be discussed in a later section. In contrast with that, the working assumption for this article is that sites are specific locations with relatively stable characteristics.

Although freezing rate and nucleation rate, as defined here, appear to be nearly the same, the difference is important and lies at the heart of basic uncertainties about heterogeneous ice nucleation. Thus, it is important to examine to what extent conditions for valid applications of Eq. (6) have been satisfied in past experiments, and whether interpretations of observation in terms of nucleation rate are justified or not. To accomplish this, freezing rate will be used to discuss the data obtained, even in cases where the authors referred to nucleation rate in their analyses. For simplicity, where the distinction is not needed, the term "rate constant" will also be utilized. Use will also be made of the quantities

$\omega=-\frac{\mathrm{d}(\ln R)}{\mathrm{d} T} \quad$ and $\quad \varepsilon=\exp (\omega \cdot \Delta T)$,

the temperature derivative of the logarithm of the rate constant, and (with $\Delta T=1^{\circ} \mathrm{C}$ ) the factor increase in $R$ over $1{ }^{\circ} \mathrm{C}$ of temperature decrease. Both quantities are independent of the absolute values of $R$ and have the same value for temperature scales in Celsius or Kelvin. In principle, these parameters are functions of temperature, but as will be seen, nearly constant values can characterize most experimental data sets. If that is the case, the parameter $\omega$ is the slope of the best fit line of the logarithm of the measured values of $R$ as a function of temperature - i.e., the constant in $R(T) \propto \exp (\omega T)$. The value of $\varepsilon$ is an easy reference to how fast the freezing rate increases with decreasing temperatures. It should be noted that for the case of uniform rate of cooling, the value of $\omega$ will be the same whether it is determined using $R(t)$ or $R^{*}(T)$.

\subsection{Nucleus spectra}

Closely related to the temperature-dependent freezing rate, $R^{*}(T)$ in Eq. (4), is the interpretation of that quantity in terms of concentrations (spectra) of nucleating particles per unit volume of the samples. Determination of the spectra is accomplished by steady cooling of numerous sample units drawn from a single bulk sample. The formulation is based on the time-independent singular model (Vali,1971; V71):

$k(T)=-\frac{1}{V \cdot N_{\mathrm{L}}} \frac{\mathrm{d} N_{\mathrm{L}}}{\mathrm{d} T}$ 
and its integral

$K(T)=\left(\ln N_{0}-\ln N_{\mathrm{L}}\right) \cdot \frac{1}{V}=-\frac{1}{V} \ln \left(1-\frac{N_{\mathrm{F}}}{N_{0}}\right)$,

where $k(T)$ is the differential active site density (differential spectrum) and $K(T)$ is the cumulative active site density (cumulative spectrum) per unit volume of water sample. As can be seen, the only formal difference between the right-hand formulas in Eqs. (4) and (8) is the inclusion of drop volume $V$. The essential difference is in the meaning attached to the nucleus spectra. The differential spectrum expresses the interpretation that the observed freezing rate is determined by the number of sites becoming active in the temperature interval considered. The cumulative spectrum is the frequency distribution per unit volume of water of particles causing nucleation at temperatures higher than $T$.

The fact that Eqs. (4) and (8) differ only by a factor $V^{-1}$ shows that for experiments with continuous cooling the temperature-dependent freezing rate $R^{*}(T)$ is a measure of the differential nucleus concentration: $R^{*}(T) \propto k(T)$. Also, the value of $\omega$ can then be computed directly from $\Delta(\ln k) / \Delta T$. Expressing nucleating ability via $k(T)$ or $K(T)$ does not assume that each site is located on a separate particle, neither is it necessary for valid evaluation of the spectra by experiment that each sample volume contain only one INP. Overlap and multiple INPs per drop are accounted for statistically in the extension of Eq. (8) to a finite difference form, as shown in Eq. (11) of V71. In practice, if the overlap leads to a saturation effect, dilution of the sample can eliminate the problem.

It is possible to change from a volume to a surface reference in Eqs. (8) and (9). The functions $k(T)$ and $K(T)$ then refer to the number of sites becoming active at $T$ per unit surface area of the INPs in the bulk sample. With known sizes of the particles, the spectra can express the surface density of sites, called "ice-active surface site density (IASSD)" in Connolly et al. (2009) and "ice nucleation surface site (INAS) density" in Hoose and Möhler (2012). The expressions by which these quantities are derived are variants of Eq. (9), with $A \cdot n_{\mathrm{s}}(T)$ equated to the cumulative spectrum $K(T) \cdot V$, which is the Poisson probability of encountering a site with activity above the given temperature in the sample unit of volume $V$ containing INPs of surface area $A$. In this paper, the $k(T)$ and $K(T)$ are used only as concentrations per unit volume of water because that reference (volume of sample drops) is the most accessible from an experimental point of view.

The underlying concept in the use of nucleus spectra to characterize freezing nucleation is that INPs contain sites, each of which can be assigned a characteristic temperature $T^{\mathrm{c}}$, and that it is the abundance of the sites with different values of $T^{\mathrm{c}}$ that is the crucial parameter for determining the potential of some material to promote ice nucleation. This is the "static" factor referred to earlier.

\subsection{The VS66 model}

time dependence introduced by the fluctuating growth of embryos is incorporated in the concept of nucleation rate and has to be accounted for in any model. The singular model and the definition of nucleus spectra in Eqs. (8) and (9) do not include this dynamic factor. An extension of the singular model by Vali and Stansbury (1966, VS66) is a qualitative formulation of the combined static and dynamic factors. Early support for the VS66 model was given by Okamoto and Yamada (1970) with observations of the solidification of liquid indium droplets. As will be presented in later parts of this article, essentially all available empirical data are consistent with this model.

Following Fletcher (1958), the view taken in VS66 was that nucleating sites can be described by a nucleation rate function $J(T)$ anchored to a characteristic temperature $T^{\mathrm{c}}$ specific to that site. The freezing temperature of a given drop is determined, with small random variations about it, by the nucleus with the highest characteristic temperature found in the drop. This model is illustrated schematically in Fig. 1c. The function $J(T)$ is shown for four nucleating sites having characteristic temperatures $T_{i}^{\mathrm{c}}, T_{j}^{\mathrm{c}}$, etc. These values correspond to the temperatures at which $J$ takes on an arbitrary value conveniently taken as $J_{T^{\mathrm{c}}}=1 \mathrm{~s}^{-1}$. Here the nucleation rate $J$ is taken to have dimension of inverse time, as it refers to a single entity and not to the volume or surface area of the nucleating material. For simplicity, Fig. 1 shows $J(T)$ having the same form for all values of $T^{\mathrm{c}}$ but this assumption could be replaced by more complex ones without altering the essence of the model. However, it is required that $J(T)$ cover a range of many orders of magnitude within a temperature interval that is small compared to the range of $T_{i}^{\mathrm{c}}$-values found in the sample.

For comparison, Fig. 1a shows the simplest stochastic assumption that a single $J(T)$ function is valid over the whole range of observations (well beyond the values of the ordinate in this graph) and for all sample units. Figure $1 \mathrm{~b}$ is a depiction of the basic form of the singular assumption for the same four sites as in Fig. 1c.

The key results in support of the VS66 model are the observations that, if cooling of a population of drops from the same sample of water is interrupted for a period of time and the temperature is held constant, the number of freezing events during that period is just a fraction of all the samples still unfrozen, and that the freezing rate decreases after cooling stops. In addition, as shown in Vali (1994; hereafter V94), if a short period of warming is introduced before holding the temperature fixed, the freezing rate drops to a very low but non-zero value. Neither the stochastic nor the singular models provide an adequate explanation for these observations.

According to VS66, the temperature at which nucleation is observed to take place in any particular experiment will differ from $T_{i}^{\mathrm{c}}$ depending on the rate of cooling and on chance. Conversely, $T_{i}^{\mathrm{c}}$ cannot be determined exactly from a single 


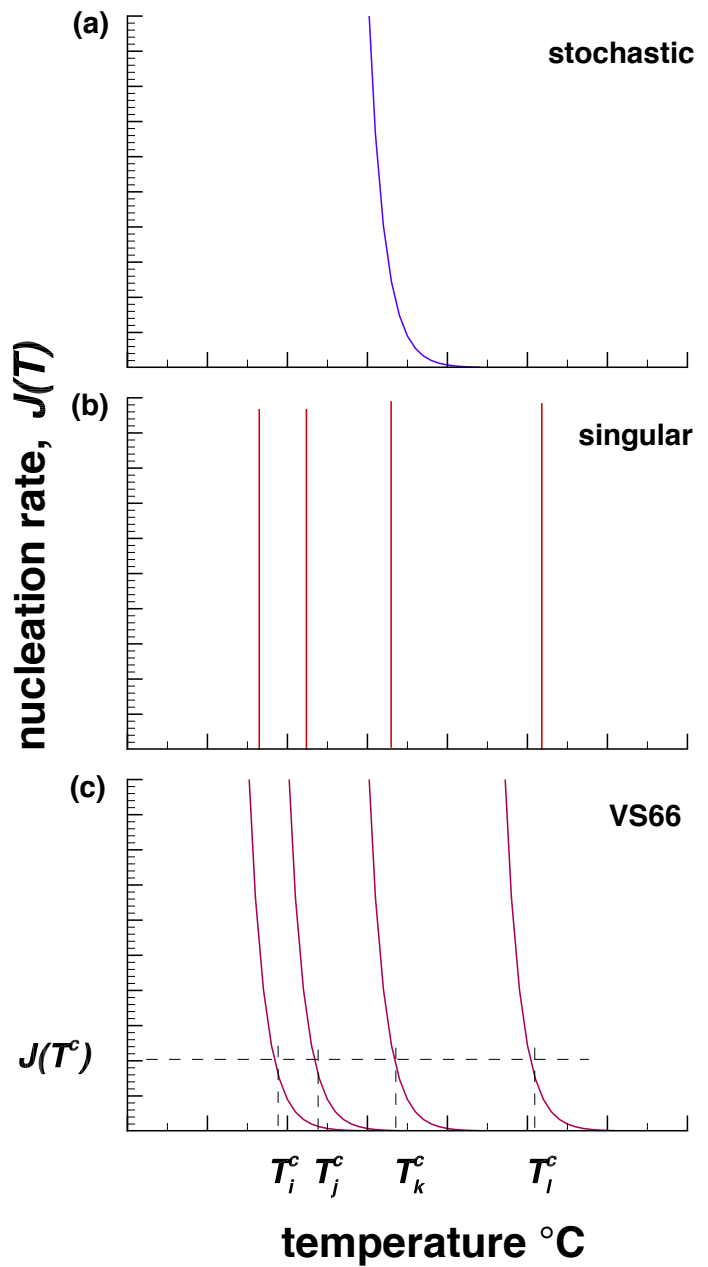

Figure 1. Schematic representation of the assumptions made about nucleation rate in the (a) stochastic, (b) singular, and (c) VS66 models.

realization of freezing. However, the observed freezing temperature in a given experiment provides a good approximation to its value. This is so, because $J(T)$ rises over many orders of magnitude over a narrow range of temperatures. For a population of sample units, the difference between the empirical $k(T)$ and the more fundamental $k\left(T^{\mathrm{c}}\right)$ is relatively small. A practical limitation is that finite temperature intervals need to be used for evaluation of $k(T)$, as in Eq. (11) of V71. Furthermore, the derived value of $k(T)$ is dependent on $w$, the rate of cooling of the sample. According to V94, $k(T)=k\left(T^{\mathrm{c}} \pm \Delta T\right)$ and $\Delta T<1^{\circ} \mathrm{C}$ for a reasonable range of $w$ values. On the average, $\Delta T$ approaches zero if the cooling rate is high, because less time is available for nucleation to take place at a temperature higher than the characteristic temperature (as it may happen if the rate of cooling is slow); this leads to lower average freezing temperatures for higher cooling rates. More detail about this point is included in Sect. 3.2.2.
For a population of sample units held simultaneously at the same temperature $T$, the number of freezing events is limited due to the fact that sample units which happen to contain the most effective nucleating site with $T_{i}^{\mathrm{c}}=T-\Delta T$ will have an increasingly lower probability to become active due to the fact that the nucleation rate decreases rapidly for increasingly positive values of $\Delta T$. The increase in the number of potential sites at a somewhat lower temperature is insufficient to offset the much lower value of $J$ for those sites at $T$. This point is also taken up again in Sect. 3.2.2.

As described in the foregoing, VS66 is a qualitative formulation. The only explicit requirement for it to be meaningful is that the spread in $T_{i}^{\mathrm{c}}$ values for the population of sites available on a substrate be larger than the range of temperatures over which $J_{T^{c}}$ covers all practically relevant values. A quantitative definition of the limits of validity of the model has not yet been attempted.

\section{Observations of heterogeneous immersion freezing}

A number of selected publications are summarized in what follows, in order to seek out what common factors can be identified. To accomplish this, reported results were utilized in the simplest possible form. The experiments reviewed are divided into two major groups: those that involved repeated freezing of the sample units and those with single tests of numerous sample units. Freezing rates, as defined in Eqs. (1) and (4), were extracted from the published data and are summarized in Fig. $2 a$ and $b$. The data are not normalized by volume or surface area except for those experiments which involved measurements with the same setup for different concentrations of the INPs. Because much of the data was derived from graphs in the published articles, the accuracy of the plots in Fig. 2 is limited. The values entered in Table 1 for $\omega$ and $\varepsilon$ are also approximate; these were derived by linear fits to the major portions of each data set. No probable error ranges were considered. The plots and derived values are sufficient for the purposes of this article and certainly do not reflect adequately the actual precision of the measurements.

\subsection{Experiments with repeated freezing cycles}

Repeated freezing of the same sample at a specific test temperature is a powerful and direct way for observing what consequences arise from the fluctuating and probabilistic nature of nucleation. Ideally the sample would contain only one nucleating site and that site would be perfectly stable. Clearly, these conditions cannot be rigorously realized. Additionally, an inherent uncertainty exists in these experiments since setting $t=0$ when the sample reaches the test temperature ignores the time spent while the sample is brought from $0^{\circ} \mathrm{C}$ to the test temperature. No definitive way has been found for making corrections for this. Additional uncertainty 
Table 1. Summary of selected experiments reviewed in the text. Abbreviations in the first column are used in the text for easy reference. If a figure number is given in the second column in addition to the referenced, it identifies the source of the data used. Experiment type in the third column is a reference to a text section. The definition of $\omega$ and of $\varepsilon$ are given in Eq. (7).

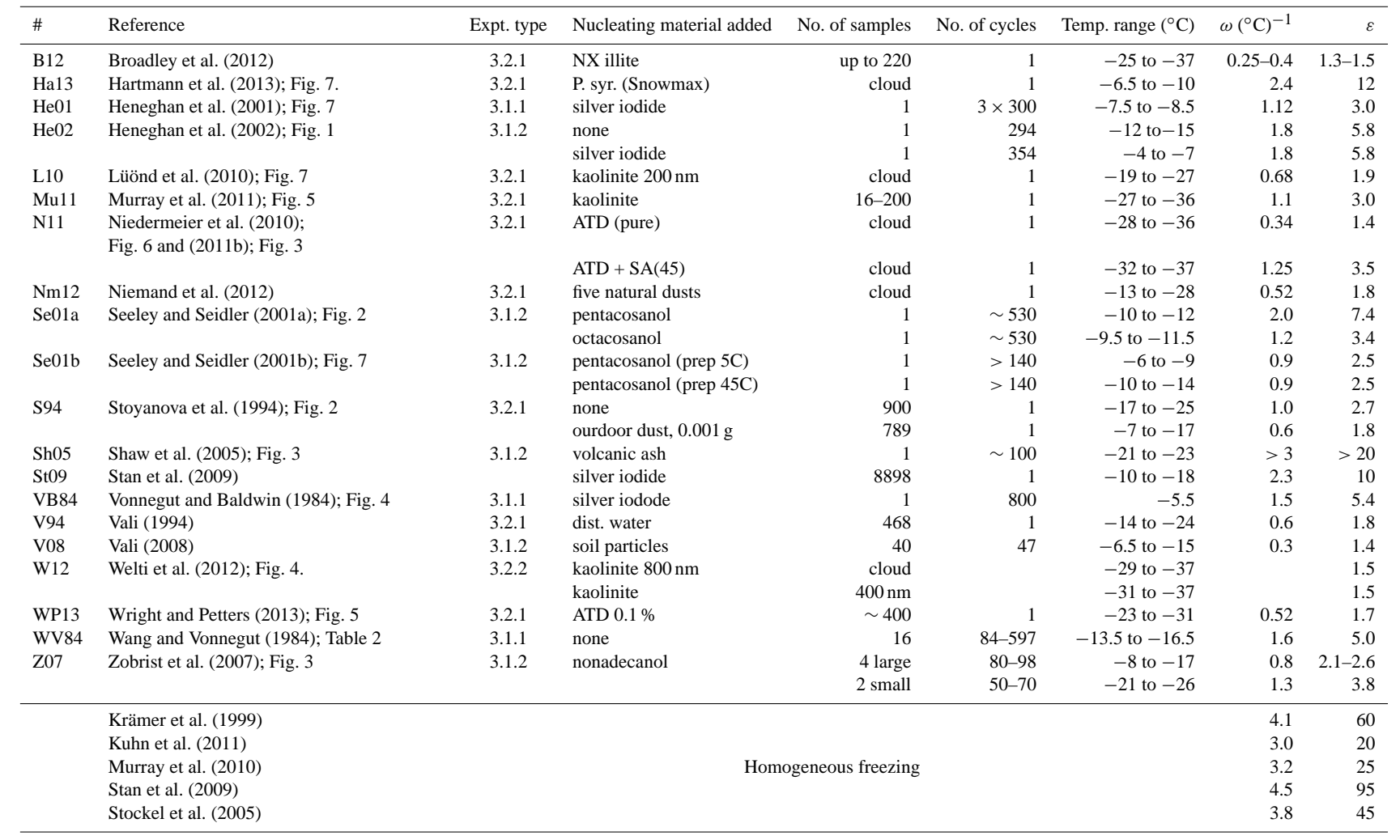

arises from the temperature gradients in the sample as it comes to thermal equilibrium with its surroundings.

Two kinds of experiments fall into this group; those using a single test temperature, with the time until freezing as the measured variable, and those in which the sample is cooled until freezing takes place and the temperature of freezing is the observed variable. Interesting differences arise from these two approaches. The main one is that experiments at constant temperatures are restricted by practical considerations to measurements of times-to-freezing that are orders of magnitude longer than the times available at given temperatures during constant cooling, and so the range of values for the measured rate constants are quite different. Large sample sizes (numbers of repetitions) can mitigate this difference.

\subsubsection{Experiments with single samples at fixed temperatures - measurements of time-to-freeze}

The definition of nucleation rate as a probability of freezing per unit time, when applied to heterogeneous nucleation, has to be considered in reference to a large number of identical nucleation sites, as stated earlier. With current knowledge about what constitutes a site, that condition cannot be fully realized. An alternative approach, at least in principle, is to observe the frequency distribution of the time until freezing in many repetitions with the same sample at the same temperature. Making that measurement at different temperatures with the same sample can lead, ideally, to the function $J(T)$.

Perhaps the earliest attempt to perform experiments of this type was that of Johnson (1948). Measurements were made with drops suspended on filaments inside a large cooled chamber. Drop temperatures could not be well stabilized with this apparatus so the only result that can be extracted from the observations is that at temperatures ranging from -15 to $-35^{\circ} \mathrm{C}$ several minutes to over an hour passed before freezing. A total of 50 observations were made. Bayardelle (1954) argued against the stochastic interpretation based on results with the repeated freezing of drops maintained at a mercuryoil interface but did not have sufficient data to draw further conclusions.

Vonnegut and Baldwin (1984; hereafter VB84) used an automated apparatus for repeated immersion of a sample into a temperature-controlled bath. The water sample was at the bottom of a U-shaped glass tube and had silver iodide particles in it. Freezing was detected by the change in conductivity of the sample. The range of times-to-freezing for 800 repetitions at $-5.5^{\circ} \mathrm{C}$ ranged from a few seconds to over $5 \mathrm{~min}$, with a reasonably good fit to the expected exponential decrease in $\mathrm{d} N_{\mathrm{L}} / \mathrm{d} t$ and hence in $N_{\mathrm{L}}$. The value 


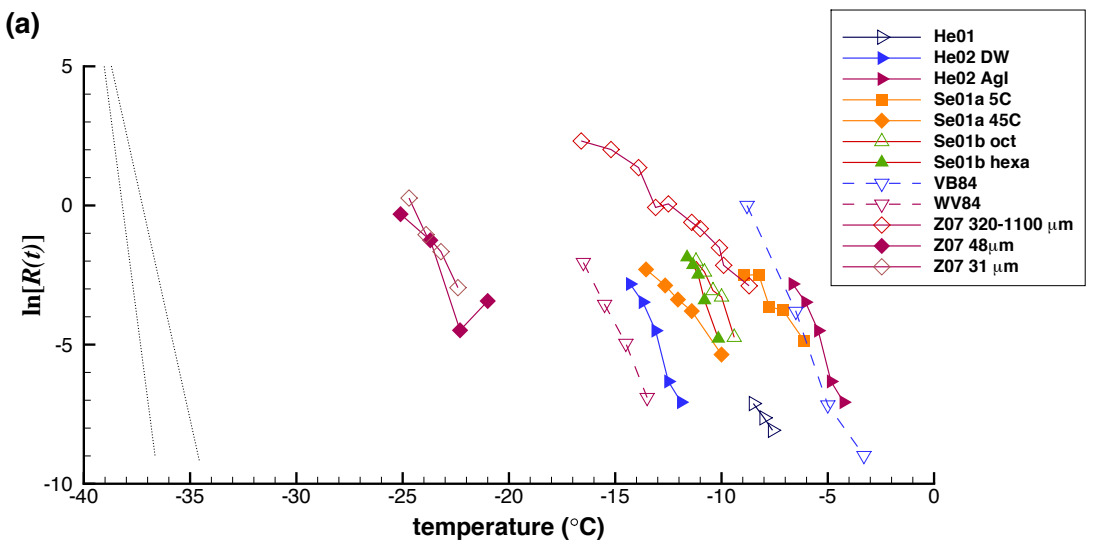

(b)

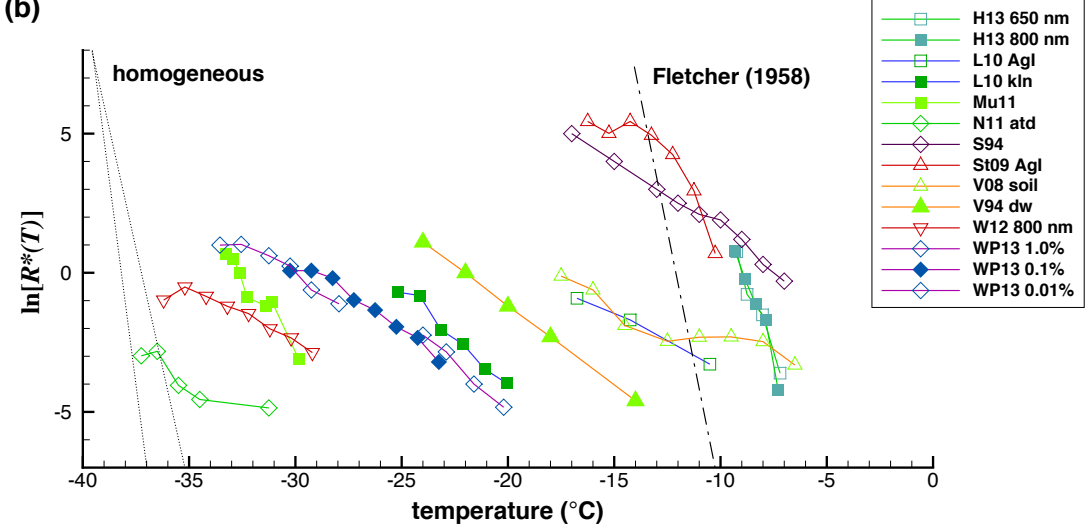

Figure 2. Measured freezing rates in different experiments. The legends in the two panels identify the sources using the abbreviations defined in the text and in the first column of Table 1. In some cases the legend has added specification of the material used in the test from the fourth column of Table 1. The upper panel shows data for the freezing rate per unit time $R(t)$, while the lower panel collects data for the freezing rate per unit temperature interval $R^{*}(T)$. The number of data points has been reduced in all cases for the sake of clarity.

of $R$ in Eq. (4) was found to increase rapidly for temperatures decreasing from -3 to $-9^{\circ} \mathrm{C}$. These facts fit the expectations. However, the presence of some systematic variations in the sequences of time-to-freeze revealed that the assumption of stability of the INP was not fully justified. Also, when re-tested after a lapse of several months the samples were found to have changed, thus confirming that the sample may have undergone minor changes even during the initial series of tests. Evaluation of the data was apparently done (not clearly stated by the authors) by counting the time to freezing, starting $8 \mathrm{~s}$ after the moment of insertion of the test tube into the chilled bath. The $8 \mathrm{~s}$ delay is quoted in Baldwin and Vonnegut (1982) as the time needed for the sample to arrive within $0.1^{\circ} \mathrm{C}$ of the bath temperature. It is unclear whether different time delays were used for data taken at different temperatures. As the authors indicated, measurable time lags (neither too short to measure reliably, nor too long to be impractical for the experiment) could be obtained only over a narrow range of temperatures for a given sample. It is not clear if all the data in the paper in fact was obtained with the same sample or with different ones (all silver iodide).
If, in spite of the complications, one applies the stochastic model, one reads from Fig. 1 of the paper $R=0.014 \mathrm{~s}^{-1}$ for $-5.5^{\circ} \mathrm{C}$, and from Fig. 4 of the paper $\omega=1.62$, and $\varepsilon=5.4$, in other words, a factor of 5.4 increase in $R$ for each degree lower temperature.

Wang and Vonnegut (1984; hereafter WV84) reported on tests with distilled water using the same apparatus as Vonnegut and Baldwin (1984). Freezing events were found to be in the range of -13.5 to $-16.5^{\circ} \mathrm{C}$. Interestingly, $\omega=1.61$ for these samples too, almost identical to the value with silver iodide.

Heneghan et al. (2001; hereafter He01) reported tests similar to those of Vonnegut (VB84, WV84). In these experiments, the sample was contained in a small tube, immersed in a bath whose temperature could be programmed to perform many cycles of cooling and heating. The volume of water was $500 \mu \mathrm{L}$ and one silver iodide crystal was introduced into the sample container. A single crystal was used in order to make it more likely that all freezing events initiate on the same site. Experiments were performed at $-7.6,-8.0$ and $-8.5^{\circ} \mathrm{C}$. The observed times-to-freezing extended up to $3 \mathrm{~h}$. 
In order to evaluate $R$, the initial $1000 \mathrm{~s}$ were ignored because fewer events were observed over that period than expected from a backward extrapolation of the exponential function derived for later times; this was ascribed by the authors to temperature lags in the sample. Randomness of the time series of freezing temperatures past the initial $1000 \mathrm{~s}$ was supported by various statistical tests. Accepting the decay rates fitted to the main segments of the data, the rate constants determined in these experiments were (as read from the published graphs) $R=3.1 \times 10^{-4}, 4.9 \times 10^{-4}$ and $8.1 \times 10^{-4} \mathrm{~s}^{-1}$ for $-7.6,-8.0$ and $-8.5^{\circ} \mathrm{C}$, respectively. These values yield $\omega=1.12$ and $\varepsilon \approx 3$. Measured values of $R$ for the same temperature are smaller than those of VB84 by factors of roughly $10^{3}$, probably due to the fact that the amount of silver iodide used in the two experiments were quite different.

\subsubsection{Experiments with single samples and steady cooling - measurements of freezing temperatures}

In the experiments of Seeley and Seidler (2001a, 2001b; hereafter Se01a and $\mathrm{Se} 01 \mathrm{~b}$ ) a drop of water $10 \mu \mathrm{L}$ in volume was supported on a prepared glass surface. Cooling was at $-3{ }^{\circ} \mathrm{C} \mathrm{min}^{-1}$ and $100-200$ cycles of freezing and melting were performed for each sample. The drops were coated by various aliphatic alcohols. In $\mathrm{Se} 01 \mathrm{a}$, the temperature to which the samples were heated between cooling runs was varied in order to gain insight into the preactivation mechanism of the crystalline films but that aspect of the experiments is not dealt with here. Freezing rates were derived by equating the fraction of runs in which the sample remained unfrozen at a given temperature with what the unfrozen fraction would be within a large number of identical samples tested simultaneously. Apparent randomness of the observed freezing temperatures with time for four of the six series of reported measurements provided justification for that assumption. In two runs, those with the most active coatings, the freezing temperatures showed a gradual decrease with time, so for these samples the freezing rates cannot be validly deduced in a direct way. For the four steady series, freezing temperatures were in the range of -6 to $-12{ }^{\circ} \mathrm{C}$ and the freezing rates for these are included in Fig. 2a and in Table 1.

Using the same apparatus as in $\mathrm{He} 01$, experiments with steady cooling were performed by Heneghan et al. (2002; hereafter He02). The sample held in a tube was cooled at a steady rate of $w=-1{ }^{\circ} \mathrm{C} \mathrm{min}^{-1}$ in hundreds of repeated cycles. Results are reported for two series of experiments, one with pure water, the other with a silver iodide crystal immersed in the sample. The fraction of runs in which the sample remained unfrozen at a given temperature was equated to what the unfrozen fraction would be within a large number of identical samples tested simultaneously. The distilled water and the silver iodide samples exhibited identical temperature dependence in spite of a nearly $8^{\circ} \mathrm{C}$ difference in the actual freezing temperatures observed. For this paper, in order to make the data directly comparable among different experiments, the rate constants were re-computed from the published plot of the fraction frozen (Fig. 1 in $\mathrm{He} 02$ ). For this, Eq. (4) was applied with $d g$ as the change in the fraction unfrozen between $T$ and $(T+\mathrm{d} T)$ and using $\mathrm{d} t=\mathrm{d} T / w$. Values of $R$ so obtained increased from $8 \times 10^{-4} \mathrm{~s}^{-1}$ to $0.25 \mathrm{~s}^{-1}$ within the interval of -12 to $-14.5^{\circ} \mathrm{C}$ for distilled water, and between -4.5 and $-7^{\circ} \mathrm{C}$ with the silver iodide crystal. The plots of the data in Fig. 2a led to $\varepsilon=5.8$. The $R$ values here obtained and plotted in Fig. 2a are different from those reported in the paper.

Zobrist et al. (2007; Z07) described another study of the nucleating activity of aliphatic alcohol coatings. Water drops supported on glass or metal surfaces were covered with nonadecanol and subjected to repeated freezing cycles with cooling rates of $-10^{\circ} \mathrm{C} \mathrm{min}^{-1}$. Freezing rates were derived by counting the frequency of freezing events per discrete temperature intervals and normalized by the water-monolayer contact area. The authors showed that the observed freezingrate vs. temperature function could be reconciled with CNT if the contact angle increased with decreasing temperatures. We will return to this point later. Data shown in Fig. 2a from this work was obtained by re-calculating the freezing rates from the number of freezing events reported in Table 1 of the paper and normalized by the square of the drop radius. From these data, $\varepsilon=2.3$ for the larger drops and $\varepsilon=3.8$ for the smaller drops.

Shaw et al. (2005; Sh05) performed repeated cycles of cooling and melting with a drop of water which had a small piece of volcanic ash placed either inside the drop or at its surface. Freezing temperatures were near $-20^{\circ} \mathrm{C}$ with the particle immersed in the drop. Freezing temperatures varied within a range of $\sim 1^{\circ} \mathrm{C}$ over 100 cycles. The very narrow range of freezing temperatures for immersion freezing (Fig. 3 in Sh05) make it difficult to extract a reliable $R^{*}(T)$ function, but appear to justify a rough estimate of $\varepsilon>20$. With the particle at the drop surface, freezing temperatures were near $-16^{\circ} \mathrm{C}$ for about $90 \%$ of the events, with variations of $<1^{\circ} \mathrm{C}$. Scattered along the time sequences, exceptionally low freezing temperatures occurred in both series of tests in a fashion similar to that found in other experiments with repeated cycles of freezing.

Hoyle et al. (2011; hereafter Ho11) reported results for 810 freezing cycles with samples containing either volcanic ash particles or ATD (Arizona test dust). In the majority of cases, the range of variation in mean freezing temperatures for externally identical samples was greater than the variation in freezing temperatures for the individual samples. Depending on dust concentration and size, freezing temperatures varied between -8 and $-20^{\circ} \mathrm{C}$. Pinti et al. $(2012$, P12) performed experiments of cycles of freezing and melting with samples of $\sim 2500 \mu \mathrm{L}$ and with different clay mineral particles in them. Freezing temperatures ranged from -5 to $-25^{\circ} \mathrm{C}$ for individual samples. In 20 repetitions, fluctuations of $\sim 1^{\circ} \mathrm{C}$ magnitude were observed in a number of cases, while other samples showed fluctuations of up to $8^{\circ} \mathrm{C}$. Both 
of these experiments are consistent with the notion of specific sites, but the support is weak because the experiments were not designed to explore that point in depth.

Even though many sample drops were used in each experiment, the results shown in Vali (2008; hereafter V08) for a soil suspension are of the same general type as others in this section, since the freezing temperatures were recorded individually for each drop and were analyzed as separate sequences. Deviations from the mean temperature for each drop were used to determine the freezing rate for a soil sample that had freezing temperatures in the range of -6.5 to $-15^{\circ} \mathrm{C}$. For 40 drops in 47 runs the values obtained were $\omega=0.9$ and $\varepsilon=2.4$, but with a significant decrease in the slope for the part of the curve corresponding to negative deviations from the mean. Ninety percent of the changes were less than $1.8^{\circ} \mathrm{C}$ in magnitude. If instead of the deviations from the mean, the freezing rate is evaluated in terms of runto-run changes in freezing temperatures the result is $\omega=1.7$ and $\varepsilon=5.6$. The cumulative frozen fraction curve vs. temperature exhibited the $\mathrm{S}$-shape that is also reported in other studies. The distribution was decomposed into two components in an attempt to separate random variations from occasional alterations of the nucleating sites. The narrower distribution, assumed to represent stochastic changes associated with the nucleation rate, was characterized by a standard deviation of $0.2^{\circ} \mathrm{C}$. As a very approximate estimate, that value corresponds to $\varepsilon \approx 10^{4}$, a value that seems too high even in comparison with homogeneous nucleation. This may indicate that the separation into two separate sources of run-torun changes was not as effective as it should be.

The most recent set of measurements with repeated freezing cycles are those of Wright and Petters (2013; hereafter WP13). Experiments were done with drops of $6 \times 10^{-5}$ to $8 \times 10^{-3} \mu \mathrm{L}$ volume (50-250 $\mu \mathrm{m}$ diameter) placed on a solid surface and coated with a hydrocarbon fluid. The drops were produced from a suspension of ATD particles. Variations in freezing temperatures over 40 repeat cycles were a combination of small fluctuations and large abrupt changes. After screening for non-random variations with an autocorrelation test, the remaining sample of roughly 200 drops (about half the original set) had a spread of $\sim 0.45^{\circ} \mathrm{C}$ in freezing temperatures and a rough estimate of $\varepsilon \approx 50$. The separation of random variations from other changes seems to have been more realistic in this work than in V08.

\subsection{Experiments with multiple samples and single tests}

This group of experiments differs from those discussed in the previous section in that each set of samples was tested only once and specific sample units (drops) were not tracked individually. Many variants of such experiments have been used. Small droplets in emulsions, droplets deposited on surfaces, and droplets introduced into a cloud chamber or formed by condensation within the chamber are the main approaches. Ideally, for purposes of evaluating the data, all drops in a given test would be the same size and would contain the same nucleant(s), but this is frequently unachievable. Reducing and controlling deviations from those requirements pose significant experimental challenges and accounting for spreads in those variables usually involves some uncertainties.

The majority of experiments with populations of sample drops examine the temperature dependence of freezing, either by sequences of tests at discrete temperatures or with steady cooling. In the first case, the observations can be evaluated in terms of $R(t)$ from Eqs. (1) or (2), or as $R^{*}(T)$ from Eq. (4) if the rate of cooling is known. Even if a timedependent freezing rate $R(t)$ is derived, the connection to nucleation rate is more questionable for these experiments than for those with single samples, since there are many different INPs involved and the homogeneity among the particles is not always assured. All of these experiments share the problem of how to account for the time period during cooling to the test temperature, that is, the time-temperature history of the sample.

Additionally, in a smaller number of experiments, the time evolution of the sample was studied at fixed temperatures or with varying cooling rates. In the following, findings from experiments focusing on temperature dependence are summarized first, then those examining time dependence are reviewed.

\subsubsection{Experiments with variable temperatures}

Independently of whether discrete test temperatures were used or steady cooling was applied, the results for this group of experiments are all presented in terms of $R^{*}(T)$ and are plotted in Fig. 2b. Where cumulative spectra were published, those have been differentiated and are given in Fig. 2b as concentrations, or site densities per degree temperature interval. For exponential spectra the values of $\omega$ and of $\varepsilon$ are the same for the cumulative and differential spectra.

Following many earlier studies, freezing rates were determined in V94 and V08 for millimeter-sized drops placed on a hydrophobic solid surface and cooled at a constant rate. Samples of distilled water and soil suspensions were investigated. The composition of the INPs in the distilled water was not examined and the soil samples were not classified by size. For distilled water, freezing rates were found to increase exponentially with decreasing temperatures between -15 and $-25^{\circ} \mathrm{C}$. The measurements yielded $\omega=0.6$ and $\varepsilon=1.8$. For a soil suspension, $\omega=0.3$ and $\varepsilon=1.4$ represent the data reasonably well even though the exponential approximation for this sample is poorer. Some of the samples produced complex spectra with pronounced peaks and valleys.

Water-in-oil emulsions and differential scanning calorimeters (DSC) have been widely used, for homogeneous nucleation studies at first and with suspended particles more recently. Since the water droplets are not of uniform size, their size distribution is convolved with the shape of the exotherm 
and the thermal properties of the instrument. Nonetheless, the fraction of sample volume frozen as a function of time can be deduced. Marcolli et al. (2007) reported on such tests with two different size fractions of ATD. Taking into account the size distribution of the particles, the particle surface area per drop was calculated. Random allocation of the particles was assumed and the number of particles per drop was taken to be proportional to drop volume. Freezing was observed to occur between -15 and $-30^{\circ} \mathrm{C}$. Freezing rate per unit surface area was deduced using a variant of Eq. (4). The results did not match the predictions of a stochastic model. Instead, two variants of the singular model approach were constructed using contact angle as a proxy for effectiveness. In one version each particle was assumed to be characterized by a single value of the contact angle, assigned from a distribution of values. In the other version, particle surfaces were characterized by a distribution of sites of different effectiveness (defined by contact angle) in proportion to their surface area. These two versions of the singular model reproduced the observations with about the same degree of precision.

Using a microfluidic apparatus, Stan et al. (2009; hereafter St09) measured the nucleation frequency for droplets of $5 \times 10^{-4} \mu \mathrm{L}$ volume $(100 \mu \mathrm{m}$ diameter $)$ cooled almost instantaneously $\left(-100^{\circ} \mathrm{Cs}^{-1}\right)$ from above $0{ }^{\circ} \mathrm{C}$ to the test temperature and then in a series of equally rapid small steps to yet lower temperatures. Results for homogeneous nucleation were close to previous data. With silver iodide particles added to the droplets, freezing temperatures were between -10 and $-18^{\circ} \mathrm{C}$. Freezing rates derived from these measurements (Fig. 9a in St09) are shown in Fig. 2b. Over the first four-degree interval, where the rate increases rapidly, $\omega=1.7$ and $\varepsilon=5.5$. These values are close to those of VB84. At lower temperatures the freezing rates increased more slowly; the same pattern was reported in V08.

In the experiments of Lüönd et al. (2010; hereafter L10) droplets were produced by condensation onto size-selected kaolinite particles and then introduced into the Zurich Ice Nucleation Chamber (ZINC). Freezing took place in the temperature range between -30 and $-35^{\circ} \mathrm{C}$. One additional experiment was conducted with silver iodide particles for which freezing occurred between -8 and $-20^{\circ} \mathrm{C}$. The fraction of droplets frozen with different final temperatures in the chamber was the primary data product. The curves shown in Fig. $2 b$ were derived from the published graphs for $200 \mu \mathrm{m}$ kaolinite particles and for silver iodide. The slope of the silver iodide curve is significantly less than for the data of S09 and VB84. Results were shown to be compatible with a distribution of active sites ( $\alpha$-pdf model) on the particle surfaces; more will be said about this later.

Murray et al. (2011, Mu11) employed a technique in which drops of aqueous suspensions of particulates are dispersed onto a glass surface, cooled at controlled rates and observed optically to detect freezing. Drop volumes ranged from $\sim 10^{-6}$ to $10^{-5} \mu \mathrm{L}$. With kaolinite particles (type KGa$1 b)$, freezing temperatures were in the range of -27 to $-35^{\circ} \mathrm{C}$. A unique freezing rate function was used to represent all the data after adjusting each experiment for the amount of INPs in proportion to their surface area (see Sect. 5.2) . The data shown in Fig. $2 b$ represent results for sample set (v) from Fig. 1 of the publication. The slope of this line is $\omega=1.1$, which yields $\varepsilon=3.0$. With the same material but with larger drops $(1 \mu \mathrm{L})$ the tests were extended to freezing temperatures as high as $-15^{\circ} \mathrm{C}$ by Herbert et al. (2014; hereafter H14), with adjustments for variations in the rate of cooling (see Sect. 3.2.2). These results show (Fig. 4a in H14) that the same value of $\omega$ is valid for the entire temperature range. A minor reservation with the new data can be noted since some of the runs taken individually appear to have shallower slopes than the combined set.

Another set of experiments using the same technique as that used in Mu11 was performed with suspensions of the mineral NX illite by Broadley et al. (2012; hereafter B12). Different concentrations of the mineral and different cooling rates were employed. The measurements yielded $\omega=0.25-$ 0.4 for the different samples (based on Fig. 6a of the publication).

A large variety of nucleation experiments has been performed with the Leipzig Aerosol Cloud Interaction Simulator (LACIS). These experiments consist of forming cloud droplets on particles of controlled size and composition, passing the cloud through a chamber held at a fixed temperature and determining by optical means the fraction of droplets frozen. This approach has the advantage of assuring a near-perfect one particle to one ice crystal correspondence. Niedermeier et al. (2010, 2011a, 2011b; collectively N11 in Fig. 2 and Table 1) report on experiments using size-sorted ATD particles with various coatings, and similar tests with pure water droplets. Freezing temperatures were in the range of -28 to $-40^{\circ} \mathrm{C}$; those lower than $-37.5^{\circ} \mathrm{C}$ were attributed to homogeneous freezing. For the pure mineral, the freezing rate increased gradually between -28 and $-36^{\circ} \mathrm{C}$ and 10 $60 \%$ of the droplets were frozen at $-36^{\circ} \mathrm{C}$. Only at $-40^{\circ} \mathrm{C}$ did the frozen fraction reach $100 \%$. For these experiments, the data shown in Table 1 and in Fig. $2 b$ were obtained by differentiating the reported fractions frozen. For ATD, the slope of the line is $\omega \approx 0.34$, so that $\varepsilon \approx 1.4$.

Hartmann et al. (2013, Ha13) used the LACIS facility to study the ice nucleating abilities of Pseudomonas syringea bacteria, and fragments of those bacteria from the commercial Snowmax product. Freezing temperatures as high as $-6^{\circ} \mathrm{C}$ were detected, so that this data set significantly extended the range that was obtained with mineral particles (preceding paragraph). Significant increases in the fraction of frozen drops exiting the chamber were found to end at $-10^{\circ} \mathrm{C}$, with only about $20 \%$ of the drops frozen. This was interpreted as being due to lack of activity in the remaining particles. Even so, measurements were obtained over two orders of magnitude of the frozen fraction. For inclusion of these data in Fig. 2b, freezing rates were calculated from the published values of the frozen fraction (from Fig. 3 of Ha13, 
for $800 \mathrm{~nm}$ size fraction) scaled to unity at the maximum observed at $-10^{\circ} \mathrm{C}$. This analysis results in $\omega \approx 2.4$, so that $\varepsilon \approx 12$. These values agree with the slope of the line fitted to data for two different sizes of particles in Fig. 7 of the paper, as well as the value of $B$ quoted in their Eq. (9). Earlier tests for the same material (Snowmax) by Wood et al. (2002) produced similar results, with the frozen fraction rising from near zero at $-6^{\circ} \mathrm{C}$ to near unity by $-9{ }^{\circ} \mathrm{C}$. The mass concentration of Snowmax was less than a tenth of that used by Ha13 and the suspension was tested directly, not aerosolized and size sorted.

Experiments performed in the AIDA (Aerosol Interaction and Dynamics in the Atmosphere) chamber produced a substantial body of data on the nucleating abilities of various materials and on the mechanisms of ice nucleation. Niemand et al. (2012; hereafter Nm12) tested five desert dust samples and found that data from all could be described reasonably well by a single $n_{\mathrm{s}}=f(T)$ function of exponential form, although the scatter about the best fit amounted to over an order of magnitude. These data (from Fig. 6 and Eq. 5 of the paper) yield $\omega=0.52$ and $\varepsilon=1.8$, valid for the temperature range of -13 to $-28^{\circ} \mathrm{C}$. This range is extended in Hoose and Möhler (2012; Fig. 13) to nearly -10 to $-35^{\circ} \mathrm{C}$ and the data are approximated by a line with $\omega=0.63$ and $\varepsilon=1.9$. The survey in Hoose and Möhler (2012) also includes results for various other minerals, bio-aerosol, bacteria and soot.

In experiments with steady cooling of samples containing $0.01,0.1$ and $1.0 \mathrm{wt} \%$ of ATD, WP13 obtained the data shown in Fig. 2b. The plotted data were derived from the published Fig. 5, with the freezing rates adjusted to the same mass concentration of ATD. As the overlap of the three segments of the data shows, the freezing rate scaled with concentration. The plot yields $\omega=0.6$ and $\varepsilon=1.8$.

\subsubsection{Observations of time dependence}

While time dependence is a factor in all the experimental methods already discussed, two types of measurements are of special relevance in this regard: (i) observing the evolution of the frozen fraction with time at a constant temperature for populations of externally identical sample units, and (ii) varying the rate of cooling for such samples. The limited number of publications involving these types of experiments are discussed in the following.

For samples held at a fixed temperature, the stochastic model ( $R_{\mathrm{T}}=$ const.) predicts that the number of samples remaining unfrozen tends to zero with time, as indicated in Eq. (3). This pattern provides a relatively easy check of the applicability of the stochastic model. For the singular model $R_{\mathrm{T}}=0$, in other words, no additional freezing events taking place after cooling stops at temperature $T$.

Contrary to both the singular and the stochastic models, freezing rates at constant temperatures were found in a number of experiments to be non-zero, but to decrease with time ( $R_{\mathrm{T}} \neq$ const.) and to have significant numbers of sample drops remain unfrozen even after extended periods of time. Fig. 5 in Vonnegut (1948) presents such a case for purified water. Other examples are given, for distilled water, in Figs. 7 and 8 in VS66 and in Fig. 2 in V94. More recent data with similar results were reported in B12. WP13 show data for two experiments, one of which extended to $16 \mathrm{~h}$. The rate of freezing clearly slowed with time in both runs. Only about a third of the sample drops froze after $16 \mathrm{~h}$, even though all drops of the same sample would have frozen with a few degrees of additional cooling.

The foregoing experiments were carried out with drops supported on solid surfaces. Welti et al. (2012; W12) reported on experiments similar to those of L12, also using kaolinite particles and varying the time of passage of the cloud droplets through the cooled chamber. Longer residence times were found to increase the fraction of frozen droplets but the increases leveled off for most temperatures so that even after extended time periods there would have been only a limited fraction of the particles producing freezing.

V94 showed the results of tests in which the samples were briefly cooled to a temperature slightly below the one where observations then followed over extended time periods. The results showed that cooling to $(T-0.5)^{\circ} \mathrm{C}$ or $(T-1.3)^{\circ} \mathrm{C}$ reduced the initial freezing rate at $T$ by about an order of magnitude. The freezing rate then decreased further with time as in experiments in which the sample was brought to $T$ without the overshoot. These results also indicate that, in general, the freezing rate is not independent of time.

The opposite result (i.e., evidence for $R_{\mathrm{T}}=$ const.) has been reported for two experiments. With equivalence assumed between many repetitions with a single sample and a single test with many samples, a good fit to Eq. (3) is shown in Fig. 4 of $\mathrm{He} 02$ for a large number of repeated cooling to $-4.9^{\circ} \mathrm{C}$ of the same silver iodide crystal that was used for the data described in Sect. 3.1.2. The rate of decrease in the number of unfrozen samples as a function of time-tofreezing, $R_{\mathrm{T}}$, was found to agree with the value of $R^{*}(T)$ determined from the experiments with steady cooling. The same type of result is shown in Fig. 3 of Mu11 for kaolinite $\mathrm{KGa}-1 \mathrm{~b}$ at temperatures between -25 and $-32^{\circ} \mathrm{C}$ and in Fig. $4 \mathrm{~b}$ of $\mathrm{H} 14$ at $-18^{\circ} \mathrm{C}$ for the same material. For these experiments as well, the decay rates were shown to agree with the values obtained with steady cooling: $R_{\mathrm{T}}=R^{*}(T)$. Some reservations with respect to the $\mathrm{H} 14$ data are warranted: it was obtained with a small sample size, the initial $40 \%$ of the sample had a significantly faster decay rate than the overall fit, and the test at $-18^{\circ} \mathrm{C}$ is within $\sim 2^{\circ} \mathrm{C}$ of the temperature where all the samples froze in tests with cooling. This latter point may be valid more generally, since even small temperature fluctuations will have a great effect in such cases. It is also worth noting that the value of $\omega$ becomes important in this regard: the higher that value is, the more critical temperature control becomes - i.e., the results become more sensitive to the precision of the apparatus used. 
For experiments with steady cooling, VS66 observed that for drops of distilled water (freezing temperatures between -15 and $-24^{\circ} \mathrm{C}$ ), variations in the rate of cooling resulted in shifts of the mean freezing temperatures of samples by

$\Delta T=-\xi \cdot \ln \left(w_{1} / w_{2}\right)$,

with $\xi=0.3$. This result has a clear intuitive interpretation: a slower cooling rate leads to higher freezing temperatures by allowing more time for embryos to reach the critical size.

The magnitude of the shift in temperatures given by Eq. (10) can be compared with what is expected on the basis of the singular and stochastic models. For the singular model (using subscript "si") $\xi_{\mathrm{si}}=0$, since there is no time dependence of the freezing temperatures. If the freezing rate of the sample can be described by an exponential function of temperature (i. e. $R=b \cdot \exp (-a T)$ ) the prediction of the stochastic model follows from Eq. (A7) in VS66. Using subscripts "st" and "obs" for the model and observed values, respectively, $\xi_{\mathrm{st}}=1 / a$ and, following from the definition and the exponential form of $R, \omega_{\mathrm{obs}}=a$. Thus, the applicability of the stochastic model to experimental results can be evaluated by comparing the best fit values of $\xi_{\text {obs }}$ with $\xi_{\text {st }}=1 / \omega_{\text {obs }}$. For the VS66 data $\xi_{\text {obs }}=0.3$ and $\xi_{\mathrm{st}}=1 / \omega_{\mathrm{obs}}=1 / 0.6=1.52$. This indicates that the observed shifts are smaller than the prediction of the stochastic model, but larger than the prediction of the singular model, as depicted in Fig. 6 of VS66.

In an early but noteworthy report, Brewer and Palmer (1951) reported no detectable change in the freezing temperatures of one drop (near $-17^{\circ} \mathrm{C}$ ) with a tenfold change in the rate of cooling. The data are not presented in sufficient detail to allow more precise evaluations. More recently, B12 reported on tests with cooling rates varying between $w=-0.8^{\circ} \mathrm{C} \mathrm{min}-1$ and $-10{ }^{\circ} \mathrm{C} \mathrm{min}^{-1}$ : for high concentrations of the NX illite mineral, shifts of 1 to $2^{\circ} \mathrm{C}$ were observed, while at low concentrations of the mineral no effect was detected. With a very extensive data set for ATD and with the cooling rate varying from -0.01 to $-5^{\circ} \mathrm{Cmin}^{-1} \mathrm{WP} 13$ found the effect to be about $0.7^{\circ} \mathrm{C}$ for a factor 10 change in cooling rate. For the WP13 data $\xi_{\text {obs }}=0.33$ and $\xi_{\text {st }}=1 / \omega_{\text {obs }}=1 / 0.52=1.92$.

Hiranuma et al. (2013) show data for illite in the temperature range of -28 to $-33{ }^{\circ} \mathrm{C}$ for two rates of cooling in the AIDA chamber. After accounting for sedimentation of the crystals, a shift was found toward colder temperatures by about $1{ }^{\circ} \mathrm{C}$ for a factor 8 increase in the rate of cooling, so that $\xi_{\text {obs }}=0.48$

The majority of the experiments just discussed indicate roughly similar magnitudes of the temperature decreases that accompany higher cooling rates, but with no clear connection established yet to factors that control the magnitudes of the shifts. Measured values of $\xi$ fall between the values expected from the singular and the stochastic models, in concert with the VS66 model.
The stochastic model was found applicable to kaolinite $\mathrm{KGa}-1 \mathrm{~b}$ by results given in H14. Using data from Mu11 with the cooling rate varied over the range of -0.1 to $-1.0^{\circ} \mathrm{C} \mathrm{min}^{-1}$, it is shown that $\xi_{\text {obs }}=\xi_{\text {st }} \approx 0.88$. A factor 10 change in the rate of cooling corresponds to a shift of $\sim 2{ }^{\circ} \mathrm{C}$ in freezing temperatures. This is almost twice the value found for other materials.

To date, KGa-1b is the only material for which the evidence for the stochastic model includes three elements: an exponential freezing rate function over a large range of temperatures, measurements of the freezing rate at constant temperatures, and the variation of freezing temperatures with changes in the rate of cooling. As argued in Mu12, the silver iodide data of $\mathrm{HO2}$ is also describable by the stochastic model; the evidence for this is only the agreement between results obtained with one cooling rate and those at one fixed temperature. Similarly, volcanic ash samples are shown in H14 to agree with the stochastic model on the basis of agreement between $\omega$ values determined from the temperature dependence of the freezing rate and from the adjustments for cooling rate that are needed to bring two data sets into agreement.

In summary, the evidence is split. Some evidence points to the applicability of the stochastic model for specific types of INPs, and some evidence contradicts it and is, qualitatively, in accord with the VS66 model. For the majority of data available to date, the dependence on the rate of cooling, $0<\xi_{\text {obs }}<\xi_{\text {st }}$ shows agreement with the VS66 prediction but no precise value for $\xi$ is predicted. Based on the VS66 model, the value of $\xi$, and the decay rate of $R(T)$ in constanttemperature tests can be expected to depend on a combination of the values of the differential site density function and the nucleation rate function $J\left(T^{\mathrm{c}}\right)$, and not only on the value of $\omega$. That distinction disappears in terms of the stochastic model because of the assumed equivalence of freezing rate and nucleation rate and hence the tests in $\mathrm{H} 14$ described in the preceding paragraph were successful in establishing the applicability of the stochastic model. With $J\left(T^{\mathrm{c}}\right)$ not known, the magnitude of the shift with cooling rate, and the freezing rate at a constant temperature, have to be determined empirically. However, if $J\left(T^{\mathrm{c}}\right)$ is a very strong function of temperature, as is known to be the case for homogeneous nucleation, than the temperature dependence of the site density function becomes the dominant factor. Future experiments can shed light on this issue. This question has also been examined in B12; the conclusions there are similar but focus on the connection with $\omega$. 


\section{Observations and model representations of freezing rates}

\subsection{Summary of observations}

The following summary focuses on the rate of change of the freezing rate with temperature, expressed by the slope $\omega$, and the factor $\varepsilon$ increase per degree of decrease in temperature. A number of patterns can be noted in Fig. 2 and Table 1:

1. There is a paucity of data for $R(t)$ (Fig. 2a) for temperatures below $-15^{\circ} \mathrm{C}$.

2. While freezing rate increases nearly exponentially with decreasing temperatures, deviations from the linear increase in Fig. 2 are quite frequent. Without full analyses of errors it is hard to weigh the potential sources of the deviations. Complex shapes of the rate functions do not contradict any fundamental law. Because of these variations in the shapes of the $R(t)$ and $R^{*}(T)$ plots, the $\omega$ and $\varepsilon$ values are imprecise representations of the data.

3. The value of $\omega$ varies from experiment to experiment but the range of variations is limited to within a factor of about 10 . This is a purely empirical result at this time.

4. Plots of data for $R(t)$ in Fig. 2a tend to be steeper than those for $R^{*}(T)$ in Fig. $2 \mathrm{~b}$.

5. Experiments in which time-to-freezing was observed for a single sample (VB84, WV84 $\mathrm{HeO1}$ and $\mathrm{He} 02$; see Sect. 3.1.1) yielded the steepest lines in Fig. 2. $(\omega \geq 1.5)$.

6. There is no clear trend in the slopes of the lines with temperature, though there is an indication for lines to be flatter in Fig. $2 b$ at lower temperatures. However, the values of $\omega$ are identical for measurements with the same apparatus of VB84 for silver iodide and of WV84 for distilled water, even though the pairs of measured freezing temperatures were roughly $8^{\circ} \mathrm{C}$ different. The same match was found by $\mathrm{He} 02$ for distilled water with and without the inclusion of a silver iodide crystal in the sample. This is a hint that perhaps the trend in slopes in Fig. $2 b$ is a result of different observational methods.

7. Significant differences are observed even among experiments with the same nucleating substance (silver iodide, ATD and others).

8. Results from the different experimental approaches discussed in Sect. 3 lead to $\omega$-values within the same degree of scatter as experiments of any particular type.

9. In a number of experiments (e.g., Z07, WP13) the freezing rate was shown to scale with the concentration of the suspended particles. For given size-distribution of the INPs, scaling the freezing rate by mass and by surface area produces equivalent results.
10. All the observed slopes are smaller (less negative) than for homogeneous freezing.

11. The constant temperature tests and the variable coolingrate tests (Sect. 3.2.2). coincide in showing that the VS66 model (Sect. 2.3) applies qualitatively to the majority of samples tested, while the stochastic model is consistent with results available for the sample KGa- $1 \mathrm{~b}$ and for the silver iodide sample tested in $\mathrm{H} 02$.

\subsection{CNT with adjustable parameters}

Difficulties were encountered in reconciling CNT with a number of empirical results. This led authors to introduce somewhat arbitrary temperature-dependent variations in selected parameters of the nucleation rate expression in CNT in order to achieve a fit.

Stoyanova et al. (1994, S94) used three different regimes with different pairs of constants in the CNT rate equation in order to match the results of their measurements. The composite function so created was specific to that one set of experimental results, and was not expected to have general validity for other materials.

Zobrist et al. (2007) found that in order to bring CNT into agreement with their observations of nucleation by a monolayer coating, the contact angle was required to vary linearly from large values at lower temperatures to smaller values closer to the melting point. They argued that this could arise from adjustments in the monolayer, leading to better fits to the ice lattice at higher temperatures.

Marcolli et al. (2007, Ma07) found the best fit to their observations with ATD suspensions could be achieved by assuming that the contact angle varies from site to site and that the number of sites increases in a specific way with increasing contact angles. They viewed this solution as a proxy for factors not clearly identified. Following the same approach, Welti et al. (2012, W12) found a good fit to their data with the CNT rate equation combined with the assumption that the contact angle has a log-normal distribution ( $\alpha$-pdf) for different particles and hence for different droplets in the cloud within the chamber.

The "soccer ball" model proposed by Niedermeier et al. (2011a) applies CNT with a prescribed variation of the contact angle. It is assumed that each sample drop contains one particle, each particle is divided into an assigned number of smooth patches, and the patches possess a contact angle randomly selected from a frequency distribution with adjustable parameters. This solution allowed various scenarios to be explored and, depending on the constants chosen, it was shown to match the predictions of either the singular or the stochastic models. It is not clear whether the authors envisaged the patches to possess the assigned properties as permanent, or at least durable features, or if the random assignment was meant to imply a random occurrence that would manifest itself differently in any one of a series of repeated tests. A modified version of the model (Niedermeier et al., 2013) 
assigns the same contact angle to all sites on a particle, the number of sites per particle is fixed, and all particles are assumed to be identical. This model is conceptually very similar to the $\alpha$-pdf model.

\subsection{Stochastic models without CNT}

A number of authors have taken the path of replacing the CNT form of the nucleation rate function and using some parametric relationship instead. This leads to formulations which use nucleation rate in the same sense as the freezing rate defined in Sect. 2.1. The main result sought in these publications is a characterization of the INPs.

The distribution of nucleating sites within the sample drops is the key consideration in the CHESS model (stoCHastic modEl of poiSSon distributed ice nuclei) developed in Ha13. All INPs - in this case fragments of bacteria - were assumed identical and the number of INPs per drops was determined based on the Poisson distribution. The probability of freezing was determined as in Eq. (9) with the concentration of sites in a drop given as the product of the number of sites, their area, the nucleation rate and time. The nucleation rate was assumed to be an exponential function of temperature with parameters retrieved from the observed freeing rates.

An exponential temperature dependence of the nucleation rate was used in $\mathrm{H} 14$ too, with an additive term in the exponent that, for the so-called multiple-component systems, is given different values for different subsets of INP effectiveness.

\subsection{Empirical approaches}

The fundamental direction taken in empirical models is to emphasize the static factor and to define the number of nucleation sites directly in terms of the temperature at which freezing is initiated by the site. In a number of publications, mostly those which aim at comparing the effectiveness of different INPs, the singular model is adopted. With the particle size and concentration per drop known, Eq. (9) is converted to a measure of site density as discussed in Sect. 5.2. Recent examples of this approach are found in Connoly et al. (2009); Niemand et al. (2012); Hoose and Möhler (2012); Murray et al. (2012, Mu12) and O'Sullivan et al. (2014).

Going beyond the definition of nucleus spectra (Sect. 2.2), the so-called time-dependent freezing rate (TDFR) formulation was developed in V94 (see also Vali and Snider, 2013). Two steps were introduced to account for time-dependent effects. One accounts for the dependence of freezing temperatures on the rate of cooling, as already indicated in Sect. 3.2.2. The second step expresses the number of freezing events when cooling is stopped relative to the freezing rate observed at the same temperature while cooling. These two parameters introduced in the TDFR method to account for time-dependent effects have been measured only for one sample so far; data are needed with different materials and with a large range of temperatures of measurements. Both the cooling-rate dependence and the additional freezing after cooling stops are relatively small effects in comparison to the strong temperature dependence found for almost all types of INPs. Nonetheless, accounting for these properties of freezing nucleation rounds out the conceptual framework for that process and evidences how the combination of static and dynamic factors work.

Many observations support the use of exponential functions of temperature for the freezing rate (VS66, V71, B12, Ha13, and others) but significantly different forms have been observed for some samples. There is no a priori reason to anticipate one functional form or another. Connolly et al. (2009) used linear and quadratic equations to describe the cumulative number of sites per unit surface area of different dust particles. The formulation of Barahona (2012) used the sum of several functions to define the activity spectrum. Such approximations are consistent with the emphasis (in Sect. 2) on freezing rate as the basis for deriving site densities.

\subsection{CNT predictions of $\omega$}

For homogeneous nucleation, reasonable agreement between theory and observation has been found by several authors (Krämer et al., 1999; Stockel et al., 2005; Stan et al., 2009; Murray et al., 2010). Even so, the different data sets correspond to different $\omega$ values in the range from 3 to 4.5 and $\varepsilon$ values from 20 to 100 . The lines shown for homogeneous nucleation in Fig. 2 bracket this range.

Adoption of the expressions for homogeneous nucleation to the heterogeneous case in forms that can be evaluated empirically has been attempted in many ways, as already discussed. Here the focus is only on the parameter $\omega$ and on the discrepancy between values derived from CNT and the observed values.

The line shown in Fig. 2 for heterogeneous nucleation follows the formulation given in Fletcher (1958). This treatment is for the nucleation rate per unit surface area and the function is anchored to a characteristic temperature of a site. The characteristic temperature is defined as the value at which $J\left(T^{\mathrm{c}}\right)=1 \mathrm{~cm}^{-2} \mathrm{~s}^{-1}$. From this formulation one can derive the slope of the $J(T)$ functions at $T^{\mathrm{c}}$. For a particle of $0.1 \mu \mathrm{m}$ equivalent diameter

$\omega=-\frac{\mathrm{d}(\ln J)}{\mathrm{d} T} \approx-\frac{46}{T^{\mathrm{c}}}$.

Using constants as given in Fletcher (1958) and corrected in Fletcher (1969) for $T^{\mathrm{c}}=-10^{\circ} \mathrm{C}$ the result is $\omega=4.6$ and $\varepsilon=100$. This value is much greater than those observed. With the dependence of the interfacial energy on temperature ignored, Eq. (11) predicts that the slope decreases at lower temperatures. That trend also can be seen by differentiating 
the CNT equation for $J(T)$, for example Eq. (11) in Mu12. Keeping the contact angle fixed, and neglecting the temperature dependence of thermodynamic parameters, the value of $\omega$ is found to decrease by a factor of about 240 between -5 and $-35^{\circ} \mathrm{C}$. Some support for this temperature dependence of $\omega$ may be seen in Fig. 2 with somewhat shallower slopes toward lower temperatures, but the pattern is far from being as strong as predicted by either method.

In all, the disagreement between observations and CNT predictions for $\omega$ is not unexpected, since the ability of CNT is also limited in predicting differences in the nucleating abilities of different materials.

\section{Discussion}

The preceding sections laid the grounds for a discussion of what can be said about the surface sites where embryo growth takes place. Quantitation of site densities and the time-dependence of nucleation have been covered, but the most difficult aspect in constructing a conceptual framework for heterogeneous freezing nucleation is how to envisage nucleation sites. Three scenarios can be considered: (i) the sites arise randomly in space and time, (ii) the sites have specific locations but are uniform in configuration and are randomly distributed on the surfaces, and (iii) the sites have specific characters which determine the temperature of their activity. Since (i) and (ii) are practically indistinguishable and both lead to a stochastic description, they will be just labeled here as the "uniform surface" scenario. The third possibility can be tested empirically; it will be called here the "specific site" scenario. In the absence of more direct knowledge about the sites at the molecular level the evidence for either scenario is, necessarily, based on indirect indicators.

\subsection{Evidence for sites}

The fact that the total surface area of the INPs per drop is important, not just the size of the particles, as predicted by classical theory, as for example in Fletcher (1958), was first shown by Edwards et al. (1962) and well confirmed by other experiments. Recent evidence for sites located on the substrate surface was obtained in experiments in which scaling with surface area was demonstrated (e.g., Z07, WP13). Li et al. (2012) came to the same conclusion based on experiments with water drops on silicone surfaces. These results establish that the probability of finding a site for nucleation at a given temperature is proportional to the total surface area of particles within a drop. Edwards and Evans (1968) showed this by actual count of the number of mono-disperse silver iodide particles within each drop and correlating that with the observed freezing temperatures.

The importance of specific sites for deposition nucleation of ice was shown quite clearly by linking ice formation to topographic features on the crystal surfaces (Bryant et al.,
1959; Fukuta and Mason, 1963) and by the repeated appearance of the ice crystals on specific locations on the substrate (Anderson and Hallett, 1976). Saturation with respect to water is required for deposition at temperatures above a value dependent on the substrate, suggesting that nucleation in these cases is initiated by freezing. Perhaps these observations can be taken to be also significant for freezing nucleation. In general, it is thought that sites are associated with surface irregularities such as cracks, dislocations, steps, spiral faults, impurities, or other discontinuities. Fletcher (1958) presented theoretical estimates for the advantage such surface features may present for embryo formation compared to flat or curved but uniform surfaces. For particles of a material, if all sizes are produced by the same dispersion process, the probability of surface features occurring on a given particle can be assumed to be proportional to the surface area of that particle. Even though limitations to this assumption may occur with some processes of particle generation, it is a good working assumption for this discussion.

The proportionality of site occurrence to surface area does not provide a distinction between the scenarios given above. A distinction can be sought via identification of the specific site scenario. That has been shown to be approachable by examining how reproducible freezing is in a large number of samples (Sect. 3.1).

It was shown in V94 that freezing temperatures of individual drops observed in one run were highly correlated with those observed in a subsequent run (correlation coefficient of 0.98) while a simulated random selection had a correlation coefficient near zero. This is in clear contradiction with all drops having the same probability of freezing. Conversely, evidence points to the fact that the drops did not contain identical nucleating sites even though all were drawn from the same bulk preparation. More results of this kind are reviewed in Sect. 3.1.2. Both V08 and WP13 diagnosed variations in the freezing temperatures of individual drops that were much smaller than the range covered by the freezing temperatures of the different drops in the sample. These tests validate the specific site scenario with nearly permanent characteristic temperatures of the sites and put bounds on the range of variations expected in particular instances about that value. Wilson and Haymet (2012) reviewed a number of experiments and arrived at the same conclusion. While it is possible that in these various tests different substances or INP sizes were included in each drop, somewhat in the manner that multiplecomponent systems are envisaged in B12, Mu12 and H14 (see next section), that is an unlikely proposition considering the large sample sizes involved in the tests.

A caveat is needed with respect to the power of re-freezing experiments to reveal the range of activities presented by the INPs involved. The run-to-run correlations of freezing temperatures become more likely to be masked by minor variability in freezing temperatures and by measurement errors the larger the value of $\omega$ is. If the range of measured freezing rates in an experiment is, say 100, the spread of freez- 
ing temperatures for $\omega=0.5$ will be $9.2{ }^{\circ} \mathrm{C}$, whereas for $\omega=2.0$ it will be only $2{ }^{\circ} \mathrm{C}$. This problem can be countered, but only to a limited extent, by increasing the number of samples and hence the range of measured freezing temperatures and freezing rates. Resolving drop-to-drop differences significantly larger than the fluctuations in observed freezing temperatures is more difficult in the latter case.

Re-freezing experiments also show that exceptionally large run-to-run changes of freezing temperature also occur. These changes are well outside the range observed over most of the test series (Sect. 3.1). A number of tentative explanations have been offered. Sh05 mentions possible movement of the particle on the surface of the drop, or movement of the drop on the supporting surface between tests. In V08 other possibilities are listed, including some interference with the ordering of an embryo on the site that was responsible for most of the events and another site of lower characteristic temperature becoming the source of nucleation. Jumps to higher temperatures may be associated with changes in the site due to stress or other reasons. Other notions similar to those dealing with pre-activation may also apply. Clearly, these types of observations need further exploration.

The experiments with monolayer coatings deserve special consideration. In $\mathrm{Se} 01 \mathrm{a}, \mathrm{Se} 01 \mathrm{~b}$ and $\mathrm{Z} 07$ the results were interpreted in terms of nucleation rates and CNT, thereby implying a stochastic process with nucleation expected to have the same probability at any point on the monolayer surface. However, a small number of exceptional (non-random) patterns were also present in these sequences and some samples exhibited clear time trends. These could be viewed as indications for specific sites existing on the monolayers in the same sense as in the preceding paragraph. Pre-activation of the coatings was reported to be required before showing nearly stable nucleating properties, adding to the likelihood that the monolayers are not simple, smooth surfaces but need some sort of ordering by ice crystals and may have discontinuities of various kinds. That the monolayer coating is not rigid but undergoes changes as the temperature is lowered was argued by Ochshorn and Cantrell (2006). In all, the monolayers may not be all that different from other surfaces in having specific nucleation sites but this is still largely speculative.

Simulations of ice nucleation have also drawn attention to the role of specific surface irregularities as possible sites by showing that smooth surfaces are less likely to have embryos form on them ( $\mathrm{Hu}$ and Michaelides, 2007; Croteau et al., 2010). On the other hand, the observations of Gurganus et al. (2011) appear to indicate no preferred location for nucleation underneath drops placed on a silicon surface. In concert with the rather weak capacity of simulations now available to predict nucleating ability, these conclusions all have to be viewed as quite tentative.

In all, the existence of specific sites has been made quite plausible by the evidence now available, supporting the VS66 model. The degree of stability of the sites needs further definition. The reproducibility of freezing temperatures of in- dividual drops in V08 and WP13 (Sect. 3.1.2) suggest that the nucleation rate $J_{T^{\mathrm{c}}}$ varies by a factor of 50 or more per degree of temperature. These estimates are not incompatible with values obtained for homogeneous nucleation, giving them some credence, but the values quoted are based on very few experiments.

\subsection{Expressions for site concentrations}

In most publications, the observed fractions of frozen samples are related to the concentration of INPs of different effectiveness via the Poisson distribution. The various formulas used to express this in the literature are equivalent to Eq. (9) in Sect. 2.2. That equation, from $\mathrm{V} 71$, has $K(T)$ equal to the probability that a sample unit of volume $V$ will contain at least one INP active at temperatures higher than $T$. Similar expressions can be written in terms of the mass, surface area or number of INPs, or in terms of any other parameter that is statistically uniform for all sample units. Frequent use is made of the substitution $n_{\mathrm{S}}(T) \cdot A=K(T) \cdot V$ in Eq. (9), so that

$f(T)=\frac{N_{\mathrm{F}}(T)}{N_{0}}=1-\exp \left[-n_{\mathrm{s}}(T) \cdot A\right]$.

The quantity $n_{\mathrm{s}}$ has been variously termed "ice nucleation active surface site (INAS) density" or "ice-active surface site density (IASSD)", but for brevity is referred to here simply as "site density". Examples of this approach are cited in Sect. 4.4. Equation (12) implies that $n_{\mathrm{s}}$ can be assumed to be a deterministic quantity, fixed by the characteristics of the INPs. This is the singular model.

In many publications, Eq. (12) is changed by equating $n_{\mathrm{S}}(T)$ to $J_{\mathrm{S}}(T) \cdot t$ with $J_{\mathrm{S}}(T)$ as the nucleation rate per unit surface area:

$f(T)=\frac{N_{\mathrm{F}}(T)}{N_{0}}=1-\exp \left[-J_{\mathrm{S}}(T) \cdot A \cdot t\right]$.

This step, either intentionally or by oversight of its implication, links Eq. (13) to the stochastic model. However, since time is fixed by the experimental arrangement in most cases, $t=$ constant, $J \cdot t$ expresses the probability of a site being found in a sample unit just as $n_{\mathrm{s}}(T)$ or $K(T)$ does, so the stochastic element reduces to one relating to the random occurrence of a site somewhere on the surface of the INP within a sample unit, not the randomness of possibly encountering a given type of site within the sample unit.

In accord with the arguments of the preceding section, the interpretation underlying Eq. (12) is that $n_{\mathrm{S}}(T)$ represents the frequency of occurrence of different sites present in the sample and not a random development realized once. In those terms, the appropriate description of laboratory results, and hence the best basis for constructing predictive models for ice nucleation in the atmosphere or in other systems starts with the measured freezing rate interpreted as the probability of freezing, directly determined by the abundance of freezing nuclei. Knowledge of the composition, sizes and surface 
areas of INPs involved in a test is now more readily available and can be used to derive information on the nucleating abilities of INPs in a sample. Hoose and Möhler (2012), Murray et al. (2012), and others have presented data in terms of the freezing rate normalized by unit area to deduce the density of active sites as a function of temperature.

It is worth noting that Eq. (12) leads to expressing site density as a cumulative number above given temperatures (i.e., as cumulative spectra). With sufficient sample size, the differential site density spectra, $k(T)$ or equivalent per unit area, can be determined. These spectra contain more revealing information about the frequency distributions of sites than the cumulative ones. If the spectra are exponential in form the difference is minimal, but there are indications in Fig. 2 that several data sets may have more complex spectra.

time dependence, the dynamic factor, need not be ignored when evaluating site densities (nucleus spectra). Normalization of the measurements to compensate for differences in the rates of cooling used in the tests improves the quality of the results. This can be seen in the results presented in H14 for the combination of data from different experiments. The basis for the normalization method described in Sect. 3.2.2 is analogous to what the stochastic model requires but with empirical values for the constant $\xi_{\text {obs. }}$. Future work may lead to quantitative relationships between $\xi_{\text {obs }}$ and other parameters characterizing the sample.

The validity of Eq. (13) in its meaning as a stochastic system was argued in Mu12, B12 and H14 for three substances (see Sect. 3.2.2) with the implication that these substances fit the uniform scenario. These substances are called singlecomponent systems in these publications and are considered pure substances. For mixtures of materials the exponent in Eq. (13) need to be obtained, in principle, as a sum of different nucleation rates weighted by the contribution of each component to the total particle surface area (multiple component stochastic, MCS, systems). Most experiments in the past are claimed to have involved mixtures of materials, whether they were done with minerals, biological materials, or others. The findings of Mu12, B12 and H14 pose the interesting question as to what the difference really may consist of between INPs for which the stochastic description applies and those for which it does not. One may speculate that the single or multiple-component distinction arises from a difference between uniform or multiple types (configuration or size) of sites being found on the same substance, not pure vs. mixed substances. That raises the further possibility that a continuity may exist in the degree of variability of site configurations in which case the stochastic system would be a limiting case of the more general one.

The contrast between the uniform surface and specific site scenarios can also be examined by looking at values of $\omega_{\text {obs }}$. For the materials fitting the uniform scenario it is somewhat higher than for other samples but the difference is less than a factor 2 (cf. Table 1). However, if Eq. (9) is invoked for the uniform surface substrates than the value of $\omega_{\text {obs }}$ is a mea- sure of the steepness of the $J_{\mathrm{S}}(T)$ function - i.e., at most a factor of 10 rise per degree. That is in disagreement with the values of $\approx 50$ estimated from re-freezing experiments (see preceding section and Sect. 3.1.2). It is not easy to envisage reasons why the rate of rise of probability of nucleation on a uniform surface would have such a weak temperature dependence in comparison with specific sites, especially since the actual temperatures of nucleation for the uniform surface materials are in the same range as those exhibiting specific sites. It would be revealing if re-freezing experiments were done with the uniform surface materials for comparison with the data obtained with samples exhibiting specific surface sites. On the other hand, there is no significant discrepancy between the values of $\omega_{\text {obs }}$ for the uniform surface samples and the results obtained with measurements of time-to-freezing (Sect. 3.1.1).

\section{Conclusions}

1. The interpretation of freezing nucleation experiments is simplified if the results are presented in terms of freezing rate (Eqs. 1, 2 or 4 in Sect. 2.1). Freezing rate is a simple empirical expression of the observations. Further meanings can be derived from the freezing rate, depending on assumptions made and measurements available regarding the INPs, leading to results expressed in terms of site density $K(T)$ or $n_{\mathrm{s}}$ from Eqs. (9) or (12). The temperature dependence of the freezing rate is usefully characterized by the parameter $\omega$ defined in Eq. (7).

2. The most direct method of measurement of nucleation rate $\left(J_{\mathrm{S}}\right.$ or $\left.J_{\mathrm{v}}\right)$ involves many repetitions of taking a single sample to the same temperature and observing the time to freezing (Sect. 3.1.1). However, these experiments are not without problems. The time spent during cooling to the test temperature need to be accounted for and no solution for this has been developed so far. Possible alterations of the sample with time are difficult to detect except by tests for the randomness of the time series. Interpretation of other empirical methods in terms of nucleation rate need detailed justification.

3. Evidence points to nucleation sites being relatively stable features and their effectiveness can be considered in terms of the temperature at which nucleation becomes highly likely (Sect. 3.1). Characteristic temperature $T^{\mathrm{c}}$ is a useful concept for that purpose, but only an approximate value can be determined experimentally (cf. Sect. 2.3) and there is at present no theory for its prediction.

4. Limitations of the stability of sites are evidenced by gradual or abrupt changes in observed freezing temperatures and by pre-activation and memory effects. Such changes are rare (Sect. 3.1) but largely unexplained. 
5. Variations in cooling rate have been shown to lead to changes in freezing temperatures that are small compared to the range of freezing temperatures usually observed (Sect. 3.2.2). In the interpretation of freezing experiments aimed at characterizing the INPs involved it can be set aside without significant degradation of the results. However, normalization to a selected value of the cooling rate for given data sets improves the value of comparisons of results from different experiments. The basis for such a normalization is expressed in Eq. (10). It gives $\xi \approx 0.3$ for two data sets (soil suspension and ADT). A larger value (0.88) is reported in $\mathrm{H} 14$ for kaolinite $\mathrm{KGa}-1 \mathrm{~b}$. The connection between values of $\xi_{\text {obs }}$ and other characteristics of given samples is not yet known.

6. The dominance of the static factors allows meaningful use of the singular model (cf. next item) but time dependence has to be accounted for in certain experiments and in the application of nucleation models under certain conditions.

7. Site density, $K(T)$ from Eq. (9), or $n_{\mathrm{S}}(T)$ derived from Eq. (12), are good representations of the freezing nucleating ability of INPs (Sect. 5.3). Evidence shows that within limited ranges these quantities are independent of particle size. The method of fragmentation and other factors in the process of particle production may influence site density and lead to different results for the same material.

8. The temperature dependence of empirically determined site densities is most usefully expressed in terms of the differential spectra $k(T)$ of Eq. (8), or as $\Delta n_{\mathrm{s}}(T) / \Delta T$ derived from Eq. (12), if the empirical data is based on large enough samples to minimize noise in these derivatives.

9. The V71 (Sect. 2.2), $\alpha$-pdf (Sect. 3.2.1), soccer-ball (Sect. 4.2), multicomponent-stochastic (Sect. 5.3), and other similar models can be viewed as different formulations applying the concept of site densities.
10. There is progress toward being able to obtain generally valid ranges of values of site densities on different materials and thus gain some predictive capacity of freezing rates for given sample volumes and INP contents (type and concentration). Laboratory techniques have advanced greatly for performing the required experiments. Theoretical underpinning for these advances will require much more detailed knowledge about the nature of nucleating sites. This type of information helps to construct models for atmospheric and other applications.

11. The patterns seen in Fig. 2 and summarized in Sect. 4.1 are largely unexplored and unexplained at this time. A relatively narrow range of slopes is also seen in Fig. 18 of Mu12, except at temperatures closest to $0^{\circ} \mathrm{C}$. It is to be expected that further studies of the dependence of $\omega$ (Eq. 7) on temperature and on INP type will lead to better understanding of freezing nucleation. If an exponential function is a good fit to some freezing-rate data, the value of $\omega$ is the same for $R(T), k(T), K(T)$ and $n_{\mathrm{S}}(T)$.

12. Prediction of the freezing rate during constant temperature conditions (Sects. 3.2.2 and 4.4) has limited empirical basis. More data of this type are needed as these observations constitute an important basis for testing the applicability of the stochastic vs. the TDFR description.

13. The uniform surface vs. specific site scenarios (Sect. 5.2) contrast the stochastic and VS66 models. Judging the applicability of the models to different materials is now based on comparisons of observed temperature dependence and time dependence with limited data (Sect. 3.2.2). Re-freezing experiments with materials exhibiting specific sites lead to higher values of $\omega_{\text {obs }}$ than is now known for materials fitting the uniform surface scenario. Repeated freezing experiments with those materials would add significant new insights. 


\section{Appendix A}

A brief summary of the origins of the stochastic and singular models are reviewed here in order to provide a historical perspective and to help illuminate the differences in viewpoint that have been a source of controversy over several decades.

Bigg (1953) used a time-dependent freezing rate description for a population of drops, and his approach was identified as a stochastic description by Marshall (1961). The model formulated by Levine (1950) and by Langham and Mason (1958) was named the singular description by Vali and Stansbury (1966, VS66), adopting a phrase from Dorsey $(1948)^{3}$.

\section{A1 Early evidence for singular freezing}

The observation that diminishing the volume of water (Sorby, 1859), or cleaning it with filtration or distillation (Meyer and Pfaff, 1935), leads to a reduction in the temperature of freezing was interpreted by Meyer and Pfaff as an indication that foreign particles are commonly responsible for the freezing of water. The lowest temperatures reached by Meyer and Pfaff without freezing was $-33^{\circ} \mathrm{C}$. That low temperature limit matched well with the observation by $\mathrm{A}$. Wegener in Greenland that fog droplets remained liquid at $-30^{\circ} \mathrm{C}$.

Significantly more detailed insight was gained from the experiments of Dorsey $(1938,1948)$. He worked with water samples of around $8000 \mu \mathrm{L}$ volume sealed in glass bulbs. Freezing was observed in the range of -3 to $-21^{\circ} \mathrm{C}$ depending on the source of water. Each bulb was observed to have a characteristic temperature of freezing, named the "spontaneous-freezing-point" of the sample. This temperature was found to be reproducible within a fraction of a degree in cycles of melting and freezing, independently of the length of time between trials, the temperature of melting, or of the rate of cooling. Periods of observation extended to many months. Erratic behavior was observed for some samples. The effects of heating and sedimentation were also examined. In the 1938 paper, Dorsey cautiously concluded that "motes" suspended in the water are responsible for determining the spontaneous-freezing-points of samples. In the much more detailed 1948 report, a greater variety of water sources and many different treatments were reported, but the conclusions of the 1938 paper were maintained.

With no reference to Dorsey's work, but bolstered by the theoretical explanation of condensation nucleation by Volmer (1939), Rau (1944) came to essentially the same conclusions as Dorsey and made more explicit reference to particles serving as nuclei. He worked with drops condensed on a metal surface and subsequently cooled. From repeated cycles of cooling and reheating he concluded that dust particles initiate freezing and that different particles posses differ-

\footnotetext{
${ }^{3}$ Dorsey (1948) referred to the location where freezing originates in a sample - whether homogeneous or heterogeneous - as a singularity.
}

ent characteristic temperatures. The drop to drop variation of freezing temperatures was described as the spectrum of nuclei with each drop freezing at the temperature corresponding to the particle with the highest characteristic temperature.

Brewer and Palmer (1951) repeated Rau's experiments with an automated apparatus specifically designed to check the claim made by Rau that freezing temperature can be lowered to $-72^{\circ} \mathrm{C}$ in some cases by many cycles of freezing and thawing. They showed that this was an artifact in Rau's work due to alcohol vapors seeping into the system. With up to 500 cycles of observations, Brewer and Palmer found that the freezing temperature remained constant within $1{ }^{\circ} \mathrm{C}$ and often within $0.25^{\circ} \mathrm{C}$ for drops of about $0.01 \mathrm{~cm}^{3}$ in volume. Changing the rate of cooling over a factor of 10 did not affect the freezing temperature either. Brewer and Palmer obtained data for only a few drops; the freezing temperatures of these were in the range of -15 to $-20^{\circ} \mathrm{C}$. In their conclusions they agree with the main claims of Rau, namely that the freezing of a sample is "due to the presence of freezing nuclei each of which acts at its own characteristic temperature", and that "there is a whole spectrum of such nuclei".

The view expressed by Dorsey, Rau and Brewer and Palmer is what became known as the singular model.

\section{A2 Early evidence for stochastic freezing}

The dependence of nucleation on time was clearly seen in the experiments of Vonnegut (1948). Water drops were placed on a metal plate and brought to a steady temperature in the range of -14 to $-18^{\circ} \mathrm{C}$. The number of frozen drops increased rapidly over the first few minutes then continued at gradually slower rates. Slight decreases in temperature corresponded to much faster rates of freezing of the drops. Vonnegut interpreted the observations as a measurement of nucleation rates.

Bigg (1953) introduced the description of the freezing of a number of small volumes of water in terms of a probability per unit time. He showed experimental results that demonstrated the dependence of the mean freezing temperature on volume and on the rate of cooling. This result can be stated as

$T_{\mathrm{m}}=2.3\left[\log \frac{V}{V_{\mathrm{o}}}+\log \frac{w_{\mathrm{o}}}{w}\right]-16.9$,

where $T_{\mathrm{m}}$ is the mean freezing temperature in ${ }^{\circ} \mathrm{C}, V$ is the volume of the samples in $\mathrm{cm}^{3}, w$ is the rate of cooling in ${ }^{\circ} \mathrm{Cmin}^{-1}$; the constants are $V_{\mathrm{o}}=1 \mathrm{~cm}^{3}$ and $w_{\mathrm{o}}=1^{\circ} \mathrm{C} \mathrm{min}^{-1}$. The range of values for $V$ were roughly six orders of magnitude (the majority of data from a smaller range of factor 50) and one order of magnitude for $w$. Bigg also showed that Eq. (A1) is consistent with a freezing probability per unit time that is proportional to an exponential function of supercooling (temperature in ${ }^{\circ} \mathrm{C}$ ):

$$
\frac{1}{N_{\mathrm{L}}} \frac{\mathrm{d} N_{\mathrm{L}}}{\mathrm{d} t}=b e^{-a T}
$$


where $N_{\mathrm{L}}$ is the number of drops not yet frozen at $T$ and $a$ and $b$ are constants. Since this relationship does not contain any dependence on the prior history of the sample it can be properly identified as a representation of a stochastic process, and Marshall (1961) attached that designation to the theory. The most important result that led Bigg to Eq. (A2) was the observed dependence of freezing temperatures on volume as expressed in Eq. (A1). Dependence on cooling rate provided an independent check on the result: a factor 10 decrease in cooling rate led to a $2^{\circ} \mathrm{C}$ higher value of $T_{\mathrm{m}}$, while Eq. (A1) predicts $2.3^{\circ} \mathrm{C}$. Based on Eqs. (A2) and (A1), the fraction $f(T)$ of a population of drops frozen when cooled at a steady rate of $w$ can be written as

$f(T)=\exp \left(-\exp \left(\ln \frac{b V}{a w}-a T\right)\right)$.

The observed shape of the frequency distribution of freezing events, normalized to a common volume according to Eq. (A1) was shown by Bigg to be in agreement with Eq. (A3).
The linear dependence of the mean (or median) freezing temperature on the logarithm of drop volume was confirmed by Langham and Mason (1958) in a repeat of Bigg's experiments. They have also shown that the temperatures vary depending on the source (purity) of the water. The logarithmic dependence on droplet size was shown to also hold for highly purified water that froze at considerably lower temperatures $\left(-35\right.$ to $\left.-42^{\circ} \mathrm{C}\right)$. On that basis Langham and Mason argued that Bigg's measurements represented heterogeneous freezing. Furthermore, they showed that the logarithmic dependence on volume can also result from the drops containing a random selection of particles whose activity is an exponential function of temperature, similarly to atmospheric particles tested in cloud chambers. Somewhat misleadingly, Pruppacher and Klett (1997; pp. 349-351) gloss over the fact that that logarithmic volume dependence is consistent with the singular model only for an exponential form of the number vs. activity function and is not a general result. This simplification led to a number of erroneous statements in later literature. 


\section{Appendix B}

Table B1. Nomenclature.

\begin{tabular}{|c|c|}
\hline$A$ & surface area of INPs in a water drop \\
\hline$f$ & fraction of number of samples frozen \\
\hline$g$ & fraction of number of samples not frozen \\
\hline$J(T)$ & $\begin{array}{l}\text { nucleation rate (probability of freezing on given site) per unit time as } \\
\text { a function of temperature }\end{array}$ \\
\hline$J_{\mathrm{S}}(t)$ & nucleation rate per unit time and per unit surface area of INPs \\
\hline$J_{\mathrm{V}}(t)$ & nucleation rate per unit time and per unit sample volume \\
\hline$k(T)$ & $\begin{array}{l}\text { differential active site density (differential spectrum): number of sites } \\
\text { active within a } 1^{\circ} \mathrm{C} \text { interval at } T \text { per unit sample volume }\end{array}$ \\
\hline$K(T)$ & $\begin{array}{l}\text { integrated active site density (cumulative spectrum): number of sites } \\
\text { active above } T \text { per unit sample volume }\end{array}$ \\
\hline$n_{\mathrm{S}}(T)$ & $\begin{array}{l}\text { surface density of sites (number per unit surface area of INPs) active } \\
\text { above } T\end{array}$ \\
\hline$N_{\mathrm{F}}$ & number of samples frozen \\
\hline$N_{\mathrm{L}}$ & number of samples not frozen \\
\hline$N_{0}$ & total number of samples in an experiment \\
\hline$R(t)$ & freezing rate per unit time $\left[\mathrm{s}^{-1}\right]$; same as $R_{\mathrm{n}}$; Eq. (1) \\
\hline$R^{*}(T)$ & freezing rate per degree temperature interval; same as $R_{\mathrm{n}}^{*}(T)$; Eq. (1) \\
\hline$R_{\mathrm{T}}(t)$ & freezing rate at a constant temperature $T$ as a function of time \\
\hline$t$ & time in seconds \\
\hline$T$ & temperature $\left[{ }^{\circ} \mathrm{C}\right]$ \\
\hline$T^{\mathrm{c}}$ & characteristic temperature for a nucleating site $\left[{ }^{\circ} \mathrm{C}\right]$ \\
\hline$V$ & volume of water drop (sample unit) \\
\hline$w$ & cooling rate \\
\hline$\omega$ & slope of $\ln R$ vs. $T$, defined in Eq. (7) \\
\hline$\varepsilon$ & factor increase in $R$ per degree, defined in Eq. (7) \\
\hline$\xi$ & $\begin{array}{l}\text { constant in Eq. (10); measure of sensitivity of freezing temperatures } \\
\text { to cooling rate }\end{array}$ \\
\hline drop & in a general sense refers to an individual sample unit of any form \\
\hline ATD & Arizona test dust \\
\hline CNT & classical nucleation theory \\
\hline INP & $\begin{array}{l}\text { ice nucleating particle - designates a particle that has been diagnosed } \\
\text { or is expected to initiate ice by a site on its surface }\end{array}$ \\
\hline
\end{tabular}


Acknowledgements. E. K. Bigg and C. E. Morris were helpful with the early definition of the focus for this paper. Two anonymous reviewers and J. S. Snider (University of Wyoming) are thanked for their valuable observations, criticisms and suggestions. An extensive exchange of ideas with T. F. Whale, R. J. Herbert and B. J. Murray (University of Leeds) led to an agreement on common nomenclature to be used in this paper and theirs (Herbert et al., 2014). This is a step toward a broader agreement on terminology related to ice nucleation which is being coordinated by the Committee on Nucleation and Atmospheric Aerosols (http://www.icnaa.org).

Edited by: M. Petters

\section{References}

Anderson, B. J. and Hallett, J.: Supersaturation and time dependence of ice nucleation from the vapor on single crystal substrates, J. Atmos. Sci., 33, 822-832, 1976.

Baldwin, M. and Vonnegut, B.: Automatic apparatus for nucleation investigations, Rev. Sci. Instrum., 53, 1911-1914, doi:10.1063/1.1136904, 1982.

Barahona, D.: On the ice nucleation spectrum, Atmos. Chem. Phys., 12, 3733-3752, doi:10.5194/acp-12-3733-2012, 2012.

Bayardelle, M.: Sur le mecanisme de la congelation de l'eau dans les nuages, CR. Hebd. Acad. Sci., 239, 988-989, 1954.

Bigg, E. K.: The supercooling of water, Proc. Phys. Soc. B, 66, 688694, 1953.

Brewer, A. W. and Palmer, H. P.: Freezing of supercooled water, Proc. Phys. Soc. B, 64, 765-773, 1951.

Broadley, S. L., Murray, B. J., Herbert, R. J., Atkinson, J. D., Dobbie, S., Malkin, T. L., Condliffe, E., and Neve, L.: Immersion mode heterogeneous ice nucleation by an illite rich powder representative of atmospheric mineral dust, Atmos. Chem. Phys., 12, 287-307, doi:10.5194/acp-12-287-2012, 2012.

Bryant, G. W., Hallett, J., and Mason, B. J.: The epitaxial growth of ice on single-crystalline substrates, J. Phys. Chem. Solids, 12, 189-195, 1959.

Connolly, P. J., Möhler, O., Field, P. R., Saathoff, H., Burgess, R., Choularton, T., and Gallagher, M.: Studies of heterogeneous freezing by three different desert dust samples, Atmos. Chem. Phys., 9, 2805-2824, doi:10.5194/acp-9-2805-2009, 2009.

Croteau, T., Bertram, A. K., and Patey, G. N.: Water Adsorption on Kaolinite Surfaces Containing Trenches, J. Phys. Chem. A, 114, 2171-2178, doi:10.1021/jp910045u, 2010.

Dorsey, N. E.: Supercooling and freezing of water, J. Res. Nat. Bur. Stand., 20, 799-808, 1938.

Dorsey, N. E.: The freezing of supercooled water, T. Am. Philos. Soc., 38, 248-328, 1948.

Edwards, G. R. and Evans, L. F.: Ice nucleation by silver iodide: III. The nature of the nucleating site, J. Atmos. Sci., 25, 249-256, 1968.

Edwards, G. R., Evans, L. F., and La Mer, V. K.: Ice nucleation by monodisperse silver iodide particles, J. Colloid Sci., 17, 749758, 1962.

Fletcher, N. H.: Size effect in heterogeneous nucleation, J. Chem. Phys., 29, 572-576, 1958.

Fletcher, N. H.: Active sites and ice crystal nucleation, J. Atmos. Sci., 26, 1266-1271, 1969.
Fukuta, N. and Mason, B. J.: Epitaxial growth of ice on organic crystals, J. Phys. Chem. Solids, 24, 715-718, 1963.

Gurganus, C., Kostinski, A. B., and Shaw, R. A.: Fast imaging of freezing drops: no preference for nucleation at the contact line, J. Phys. Chem. Lett., 2, 1449-1454, doi:10.1021/jz2004528, 2011.

Hartmann, S., Augustin, S., Clauss, T., Wex, H., Šantl-Temkiv, T., Voigtländer, J., Niedermeier, D., and Stratmann, F.: Immersion freezing of ice nucleation active protein complexes, Atmos. Chem. Phys., 13, 5751-5766, doi:10.5194/acp-13-57512013, 2013.

Heneghan, A. F., Wilson, P. W., Wang, G. M., and Haymet, A. D. J.: Liquid-to-crystal nucleation: automated lag-time apparatus to study supercooled liquids, J. Chem. Phys., 115, 7599-7608, doi:10.1063/1.1407290, 2001.

Heneghan, A. F., Wilson, P. W., and Haymet, A. D. J.: Heterogeneous nucleation of supercooled water, and the effect of an added catalyst, P. Natl. Acad. Sci. USA, 99, 9631-9634, 2002.

Herbert, R. J., Murray, B. J., Whale, T. F., Dobbie, S. J., and Atkinson, J. D.: Representing time-dependent freezing behaviour in immersion mode ice nucleation, Atmos. Chem. Phys. Discuss., 14, 1399-1442, doi:10.5194/acpd-14-1399-2014, 2014.

Hiranuma, N., Möhler, O., Wex, H., Kulkarni, G., Boose, Y., Bundke, U., Cziczo, D. J., Danielczok, A., Ebert, M., Garimella, S., Hoffmann, N., Höhler, K., Kanji, Z. A., Kiselev, A., Raddatz, M., and Stetzer, O.: Immerion freezing of clay minerals and bacterial ice nuclei, in: Nucleation and Atmospheric Aerosols, 19th International Conference, edited by: DeMott, P. J. and O'Dowd, C. D., AIP Publishing, Melville, New York, 914-917, 2013.

Hoose, C. and Möhler, O.: Heterogeneous ice nucleation on atmospheric aerosols: a review of results from laboratory experiments, Atmos. Chem. Phys., 12, 9817-9854, doi:10.5194/acp-12-98172012, 2012.

Hoyle, C. R., Pinti, V., Welti, A., Zobrist, B., Marcolli, C., Luo, B., Höskuldsson, Á., Mattsson, H. B., Stetzer, O., Thorsteinsson, T., Larsen, G., and Peter, T.: Ice nucleation properties of volcanic ash from Eyjafjallajökull, Atmos. Chem. Phys., 11, 9911-9926, doi:10.5194/acp-11-9911-2011, 2011.

$\mathrm{Hu}, \mathrm{X} . \mathrm{L}$. and Michaelides, A.: Ice formation on kaolinite: lattice match or amphoterism?, Surf. Sci., 601, 5378-5381, doi:10.1016/j.susc.2007.09.012, 2007.

Johnson, J. C.: Study of the freezing temperatures of supercoloid water with special reference to drops having diameters from 150 to 1700 microns, Ph.D. Thesis, Mass. Inst. Techn., 90 pp., 1948.

Krämer, B., Hübner, O., Woste, L., Leisner, T., Schwell, M., Ruhl, E., and Baumgartel, H.: Homogeneous nucleation rates of supercooled water measured in single levitated microdroplets, J. Chem. Phys., 111, 6521-6527, 1999.

Kuhn, T., Earle, M. E., Khalizov, A. F., and Sloan, J. J.: Size dependence of volume and surface nucleation rates for homogeneous freezing of supercooled water droplets, Atmos. Chem. Phys., 11, 2853-2861, doi:10.5194/acp-11-2853-2011, 2011.

Langham, E. J. and Mason, B. J.: The heterogeneous and homogeneous nucleation of supercooled water, Philos. Roy. Soc. A, 247, 493-505, 1958.

Levine, J.: Statistical explanation of spontaneous freezing of water droplets, Natl. Adv. Comm. Aeronautics, Techn. Note 2234, Washington D.C., USA, 1-27, 1950. 
Li, K., Xu, S., Shi, W., He, M., Li, H., Li, S., Zhou, X., and Wang, J.: Investigating the effects of solid surfaces on ice nucleation, Langmuir, 28, 10749-10754, 2012.

Lüönd, F., Stetzer, O., Welti, A., and Lohmann, U.: Experimental study on the ice nucleation ability of size-selected kaolinite particles in the immersion mode, J. Geophys. Res.-Atmos., 115, D14201, doi:10.1029/2009JD012959, 2010.

Marcolli, C., Gedamke, S., Peter, T., and Zobrist, B.: Efficiency of immersion mode ice nucleation on surrogates of mineral dust, Atmos. Chem. Phys., 7, 5081-5091, doi:10.5194/acp-7-50812007, 2007.

Marshall, J. S.: Heterogeneous nucleations is a stochastic process, Nubila, 4, 29-32, 1961.

Meyer, V. J., and Pfaff, W.: Zur Kenntnis der Kristallisation von Schmelzen, II, Z. Anorg. Allgem. Chem., 222, 382-388, 1935.

Murray, B. J., Broadley, S. L., Wilson, T. W., Bull, S. J., Wills, R. H., Christenson, H. K., and Murray, E. J.: Kinetics of the homogeneous freezing of water, Phys. Chem. Chem. Phys., 12, 1038010387, doi:10.1039/c003297b, 2010.

Murray, B. J., Broadley, S. L., Wilson, T. W., Atkinson, J. D., and Wills, R. H.: Heterogeneous freezing of water droplets containing kaolinite particles, Atmos. Chem. Phys., 11, 4191-4207, doi:10.5194/acp-11-4191-2011, 2011.

Murray, B. J., O’Sullivan, D., Atkinson, J. D., and Webb, M. E.: Ice nucleation by particles immersed in supercooled cloud droplets, Chem. Soc. Rev., 41, 6519-6554, doi:10.1039/c2cs35200a, 2012.

Niedermeier, D., Hartmann, S., Shaw, R. A., Covert, D., Mentel, T. F., Schneider, J., Poulain, L., Reitz, P., Spindler, C., Clauss, T., Kiselev, A., Hallbauer, E., Wex, H., Mildenberger, K., and Stratmann, F.: Heterogeneous freezing of droplets with immersed mineral dust particles - measurements and parameterization, Atmos. Chem. Phys., 10, 3601-3614, doi:10.5194/acp-10-36012010, 2010.

Niedermeier, D., Hartmann, S., Clauss, T., Wex, H., Kiselev, A., Sullivan, R. C., DeMott, P. J., Petters, M. D., Reitz, P., Schneider, J., Mikhailov, E., Sierau, B., Stetzer, O., Reimann, B., Bundke, U., Shaw, R. A., Buchholz, A., Mentel, T. F., and Stratmann, F.: Experimental study of the role of physicochemical surface processing on the IN ability of mineral dust particles, Atmos. Chem. Phys., 11, 11131-11144, doi:10.5194/acp11-11131-2011, 2011a.

Niedermeier, D., Shaw, R. A., Hartmann, S., Wex, H., Clauss, T., Voigtländer, J., and Stratmann, F.: Heterogeneous ice nucleation: exploring the transition from stochastic to singular freezing behavior, Atmos. Chem. Phys., 11, 8767-8775, doi:10.5194/acp11-8767-2011, 2011b.

Niedermeier, D., Ervens, B., Clauss, T., Voigtländer, J., Wex, H., Hartmann, S., and Stratmann, F.: A computationally-efficient description of heterogeneous freezing: a simplified version of the soccer ball model, Geophys. Res. Lett., 2013, GL058684, doi:10.1002/2013GL058684, 2013.

Niemand, M., Möhler, O., Vogel, B., Vogel, H., Hoose, C., Connolly, P., Klein, H., Bingemer, H., DeMott, P., Skrotzki, J., and Leisner, T.: A particle-surface-area-based parameterization of immersion freezing on desert dust particles, J. Atmos. Sci., 69, 3077-3092, doi:10.1175/jas-d-11-0249.1, 2012.
Ochshorn, E. and Cantrell, W.: Towards understanding ice nucleation by long chain alcohols, J. Chem. Phys., 124, 054714, doi:10.1063/1.2166368, 2006.

Okamoto, T. and Yamada, M.: Time-Dependence of the Heterogeneous Nucleation of Supercooled Liquid Indium Droplets, Memoirs of the Institute of Scientific and Industrial Research Osaka University, 27, 69-75, 1970.

O’Sullivan, D., Murray, B. J., Malkin, T. L., Whale, T. F., Umo, N. S., Atkinson, J. D., Price, H. C., Baustian, K. J., Browse, J., and Webb, M. E.: Ice nucleation by fertile soil dusts: relative importance of mineral and biogenic components, Atmos. Chem. Phys., 14, 1853-1867, doi:10.5194/acp-14-1853-2014, 2014.

Pinti, V., Marcolli, C., Zobrist, B., Hoyle, C. R., and Peter, T.: Ice nucleation efficiency of clay minerals in the immersion mode, Atmos. Chem. Phys., 12, 5859-5878, doi:10.5194/acp-12-58592012, 2012.

Pruppacher, H. R. and Klett, J. D.: Microphysics of Clouds and Precipitation, 2nd Edn., Kluwer Academic Publishers, Dordrecht, Boston, London, 954 pp., 1997.

Rau, W.: Gefriervorgänge des Wassers bei tiefen Temperaturen, Akad. Luftfahrforsch., 8, 65-84, 1944.

Seeley, L. H. and Seidler, G. T.: Preactivation in the nucleation of ice by Langmuir films of aliphatic alcohols, J. Chem. Phys., 114, 10464-10470, doi:10.1063/1.1375151, 2001a.

Seeley, L. H. and Seidler, G. T.: Two-dimensional nucleation of ice from supercooled water, Phys. Rev. Lett., 87, 055702 , doi:10.1103/PhysRevLett.87.055702, 2001b.

Shaw, R. A., Durant, A. J., and Mi, Y.: Heterogeneous surface crystallization observed in undercooled water, J. Phys. Chem. B, 109 9865-9868, 2005.

Sorby, H. C.: On the freezing-point of water in capillary tubes, Phil. Mag., 4, 104-108, 1859.

Stan, C. A., Schneider, G. F., Shevkoplyas, S. S., Hashimoto, M., Ibanescu, M., Wiley, B. J., and Whitesides, G. M.: A microfluidic apparatus for the study of ice nucleation in supercooled water drops, Lab on a Chip, Roy. Soc. Ch., 9, 2293-2305, doi:10.1039/b906198c, 2009.

Stockel, P., Weidinger, I. M., Baumgartel, H., and Leisner, T.: Rates of homogeneous ice nucleation in levitated $\mathrm{H}_{2} \mathrm{O}$ and $\mathrm{D}_{2} \mathrm{O}$ droplets, J. Phys. Chem. A, 109, 2540-2546, doi:10.1021/jp047665y, 2005.

Stoyanova, V., Kashchiev, D., and Kupenova, T.: Freezing of water froplets seeded with atmospheric aerosols and ice nucleation activity of the aerosols, J. Aerosol Sci., 25, 867-877, 1994.

Vali, G.: Quantitative evaluation of experimental results on the heterogeneous freezing nucleation of supercooled liquids, J. Atmos. Sci., 28, 402-409, 1971.

Vali, G.: Freezing rate due to heterogeneous nucleation, J. Atmos. Sci., 51, 1843-1856, 1994.

Vali, G.: Repeatability and randomness in heterogeneous freezing nucleation, Atmos. Chem. Phys., 8, 5017-5031, doi:10.5194/acp-8-5017-2008, 2008.

Vali, G. and Snider, J. R.: Time and temperature dependence of freezing nucleation in a cloud parcel model, in Nucleation and Atmospheric Aerosols, 19th International Conference, edited by: DeMott, P. J. and O'Dowd, C. D., AIP Publishing, Melville, New York, 863-865, 2013. 
Vali, G. and Stansbury, E. J.: Time dependent characteristics of the heterogeneous nucleation of ice, Can. J. Phys., 44, 477-502, 1966.

Volmer, M.: Kinetik der Phasenbildung, Steinkopff, Dresden u. Stuttgart, Germany, 220 pp., 1939.

Vonnegut, B.: Variation with temperature of the nucleation rate of supercooled liquid tin and water drops, J. Colloid Sci., 3, 563$569,1948$.

Vonnegut, B. and Baldwin, M.: Repeated nucleation of a supercooled water sample that contains silver iodide particles, J. Appl. Meteorol., 23, 486-490, 1984.

Wang, M. K. and Vonnegut, B.: Repeated nucleation of supercooled water sample, J. Rech. Atmos., 18, 23-29, 1984.

Welti, A., Lüönd, F., Kanji, Z. A., Stetzer, O., and Lohmann, U.: Time dependence of immersion freezing: an experimental study on size selected kaolinite particles, Atmos. Chem. Phys., 12, 9893-9907, doi:10.5194/acp-12-9893-2012, 2012.
Wilson, P. W. and Haymet, A. D. J.: The spread of sucleation temperatures of a sample of supercooled liquid is independent of the average nucleation temperature, J. Phys. Chem. B., 116, 1347213475, doi:10.1021/jp308177b, 2012.

Wood, S. E., Baker, M. B., and Swanson, B. D.: Instrument for studies of homogeneous and heterogeneous ice nucleation in freefalling supercooled water droplets, Rev. Sci. Instrum., 73, 39883996, 2002.

Wright, T. P. and Petters, M. D.: The role of time in heterogeneous freezing nucleation, J. Geophys. Res.-Atmos., 118, 3731-3743, doi:10.1002/jgrd.50365, 2013.

Zobrist, B., Koop, T., Luo, B. P., Marcolli, C., and Peter, T.: Heterogeneous ice nucleation rate coefficient of water droplets coated by a nonadecanol monolayer, J. Phys. Chem. C, 111, 2149-2155, 2007. 